\title{
DISCRIMINACIÓN SALARIAL EN EL MERCADO DE TRABAJO ESPAÑOL CON ESPECIAL REFERENCIA AL CASO DE ANDALUCÍA
}

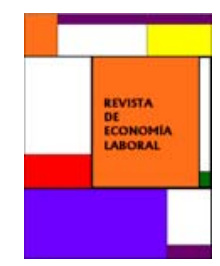

\author{
José Ignacio García Pérez*, Lola Morales López ${ }^{\dagger, 1}$ \\ *Universidad Pablo de Olavide, FEDEA, Centro de Estudios Andaluces, ${ }^{\dagger}$ BBVA
}

Recibido el 8 de octubre de 2008; aceptado el 15 de junio de 2009

\section{Resumen}

En este trabajo se analizan los factores que contribuyen a explicar las diferencias salariales entre hombres y mujeres. Utilizamos una variante de la descomposición de Oaxaca-Blinder que tiene en cuenta la censura de los datos salariales utilizados y se estiman ecuaciones de salarios suponiendo que la varianza no es constante sino dependiente de distintas caracteristicas individuales. Utilizamos datos administrativos de la Seguridad Social. Aproximadamente un $83 \%$ de las diferencias salariales medias, 17.3 puntos porcentuales, pueden deberse a la discriminación. La cualificación parece ser muy importante para explicar dicha discriminación. Con respecto a Andalucía, se encuentra una discriminación en torno al 72.5\% de los 17.7 puntos de diferencia salarial.

Palabras clave: Discriminación salarial, descomposición de Oaxaca-Blinder, modelos censurados, Andalucía.

Clasificación JEL: J16, J31, J71, C34.

\begin{abstract}
In this article, we analyze what factors contribute to explain wage differences between men and women. We apply a variant of the Oaxaca-Blinder's Decomposition Method in which the problem of censored data is taken into account. Moreover, we suppose that the wage variance depend also of different individual characteristics. Using these methods and administrative data from the Spanish Social Security, the results show that $83 \%$ of the estimated wage differences, 17.3 percentage points, is due to gender discrimination. Moreover, we find that the skills required for the job are a very important variable in order to explain wage discrimination. In the case of Andalusia, gender discrimination is about $72.5 \%$ of the 17.7 percentage points of estimated wage differences.

Key words: Wage discrimination, Oaxaca-Blinder decomposition, censored models, Andalusia.
\end{abstract}

JEL Classification: J16, J31, J71, C34.

\footnotetext{
${ }^{1}$ Autora para correspondencia: md.morales.lopez@grupobbva.com. Los autores agradecen los comentarios recibidos tanto del editor como de un evaluador anónimo. Asimismo agradecen la financiación recibida a través del proyecto SEJ2006-04803/ECON. Las opiniones y análisis de este artículo son responsabilidad de los autores y no coinciden necesariamente con las de las instituciones a las que pertenecen. Cualquier error que subsista en el texto es de nuestra entera responsabilidad.
}

(C) Revista de Economía Laboral 


\section{Introducción}

La mayoría de los indicadores que comúnmente se utilizan para medir la situación del mercado laboral son desfavorables para las mujeres: éstas tienen una menor presencia en el mercado laboral, están excluidas de algunas ocupaciones (Dolado et al., 2004), tienen una mayor representación en los empleos temporales, sufren mayores niveles de desempleo y cobran salarios inferiores a los hombres (García et al., 2001). Es en este último punto, la discriminación salarial entre hombres y mujeres, donde se centra el presente trabajo.

La literatura económica normalmente habla de discriminación salarial cuando dos trabajadores con idénticas potencialidades (en esencia misma productividad marginal del trabajo) cobran salarios distintos por razones ajenas al ámbito económico, es decir, cuando las diferencias salariales no pueden explicarse por diferencias objetivas, como pueden ser la edad, el nivel educativo, la antigüedad en la empresa, etc. Ante este hecho, la pregunta fundamental que los interesados en el tema se han hecho es si las diferencias salariales observadas entre hombres y mujeres reflejan discriminación en contra de las mujeres y en caso afirmativo, se busca estudiar en qué medida y a qué características responden. Esto implica poder controlar de la mejor manera posible los factores explicativos de la productividad de los individuos y sus diferencias en preferencias de cara a la explicación de su salario, para poder interpretar así cualquier residuo no explicado por dichas medidas como un indicador de discriminación.

Aunque se han usado muchas técnicas para analizar la discriminación, la que se utiliza más comúnmente es el método de descomposición de Oaxaca (1973) o bien generalizaciones de ésta tales como la de Brown (1980) o la de Neumark (1988). Este método permite descomponer, a partir de la estimación de ecuaciones de salarios, las diferencias salariales en dos componentes: uno primero que recoge las diferencias debidas a características observables entre los individuos, y que por tanto no podemos achacar a discriminación por parte del mercado hacia uno de los sexos, y otro segundo donde se imputa la existencia de discriminación en base a las diferencias en la retribución de cada una de estas características.

Ahora bien, para poder realizar las estimaciones de salarios por género (usando las conocidas ecuaciones de Mincer) necesitamos disponer de abundante información sobre las características de los trabajadores, tal y como Aláez y Ullibarri (2000) señalan. Esto hace que la mayor o menor publicación de trabajos relativos a este tema venga marcado por la disponibilidad de bases de datos adecuadas. Por ello, nuestro trabajo 
pretende completar los estudios realizados anteriormente sobre el tema, con una base de datos administrativa en lugar de con una encuesta como se hacía en la mayoría de trabajos anteriores. La base de datos utilizada será una muestra del fichero de afiliados a la Seguridad Social para el período 1998-2003. El hecho de que nuestra base de datos tenga una medida salarial sujeta a doble censura ${ }^{2}$ es tenido en cuenta en nuestro análisis empírico. Así, a la hora de realizar el análisis de la descomposición al estilo Oaxaca usamos una variante de la misma que nos permite analizar qué parte de las diferencias es debida no sólo a la diferencia en las medias de las características sino también en la varianza de su distribución entre la muestra.

Sin embargo, el principal atractivo de nuestra base de datos es que posee información suficiente para realizar el estudio centrándonos en una comunidad autónoma española de especial interés, Andalucía, que se comparará en sus resultados con los obtenidos para el conjunto de España. Son pocos los estudios realizados a nivel de Andalucía a pesar de ser una de las comunidades autónomas cuyo mercado de trabajo merece un estudio diferenciado al mostrar no solo salarios muy distintos, inferiores, a los del resto del Estado, sino tasas de paro y temporalidad claramente por encima de la media nacional. De esta manera, este trabajo tiene un doble interés. En primer lugar, confirmar si existe discriminación salarial en España usando una base de datos diferente y con información muy pormenorizada y fidedigna basada en datos administrativos. $\mathrm{Y}$ en segundo lugar estudiar, usando esa misma base de datos, estudiamos que ocurre en el caso andaluz; si existe o no discriminación (como Aláez y Ullibarri (2000) afirman) y cómo es comparada con la observada a nivel nacional. Ya que son pocos los estudios realizados a nivel regional sobre el tema, podremos con nuestro trabajo aportar evidencia adicional a la recogida en la literatura sobre el tema. Así, encontramos, en línea con los resultados obtenidos por Aláez y Ullibarri (2000), una mayor discriminación en España que en Andalucía. Concretamente, de las diferencias salariales observadas entre hombres y mujeres, que giran en torno al $17-18 \%$, un mayor porcentaje se atribuye a discriminación en España que en Andalucía.

El resto del trabajo se organiza de la siguiente manera. En la siguiente sección haremos un breve repaso de las distintas teóricas sobre la discriminación y de los trabajos empíricos realizados sobre el tema en España. En la sección 3 se comentan en detalle los datos utilizados en el trabajo analizando en primer lugar la base de datos y posteriormente

\footnotetext{
${ }^{2}$ Esta censura se debe a la existencia de una base de cotización mínima y máxima que limita la
} medida observada del salario. 
haciendo un análisis de las variables de dicha base usadas en nuestro estudio. La sección 4 se dedica a la descripción de las técnicas econométricas utilizadas. Por último en las secciones 5 y 6 se presentan los resultados y las conclusiones más relevantes.

\section{Literatura teórica y antecedentes.}

Las diferencias por género en el mercado de trabajo han estado siempre presentes en el análisis económico. No han sido pocos los intentos que se han hecho, desde que G. Becker en 1957 realizara su estudio pionero sobre discriminación, con el objetivo de analizar las diferencias salariares entre hombres y mujeres. Este tema ha suscitado desde siempre un enorme interés no solamente entre los investigadores del mercado de trabajo, sino también entre otras áreas de las ciencias sociales.

Existen distintos enfoques teóricos relativos a la discriminación laboral: la teoría del gusto por la discriminación (Becker, 1957), la teoría de la discriminación estadística (Aigner y Cain, 1977), así como la que defiende la hipótesis de la concentración (Bergmann, 1986).

Becker (1957) propone un modelo para explicar la discriminación salarial basado en las preferencias de los empleadores. Un empleador tiene gusto por la discriminación cuando actúa como si estuviera dispuesto a pagar algo relacionado con ciertas personas en lugar de con otras. Este autor también defiende en un artículo posterior (Becker, 1975) que una parte de las diferencias salariales por género viene explicada por las diferencias en capital humano entre hombres y mujeres (entendiendo capital humano en el sentido amplio, no sólo formación reglada sino aprendizaje en los trabajos desarrollados, etc.). De esta manera, dado que las mujeres interrumpen más su vida laboral tendrán un capital humano que se deprecia más, y esto podría ayudar a explicar las diferencias salarias observadas que no se deberían, por tanto, a discriminación.

La teoría de la discriminación estadística defiende que los trabajadores no son "discriminados" por prejuicios a la hora de contratar sino porque existen asimetrías de información. Bajo estos modelos, el salario se obtiene asumiendo que todo empleador que maximice su utilidad (costes-beneficios) preferirá contratar a aquellas personas con la máxima productividad esperada de acuerdo a información limitada que posee. Así, si la pertenencia a un grupo proporciona la información sobre la persona y su productividad (e.g., en el caso de las mujeres la información estadística afirma que la probabilidad de las mujeres de interrumpir su vida laboral es mayor que la de los hombres) el empleador maximizara su utilidad teniendo en cuenta esto. 
Bergmann (1986) sostiene que la concentración de las mujeres en unas pocas ocupaciones a causa de la discriminación lleva a la disminución de los salarios de todos los individuos en dichas ocupaciones. Por tanto, puede ser más ocupacional que salarial la discriminación que se manifieste en el Mercado de Trabajo hacia las mujeres.

Teniendo en cuenta estos distintos enfoques teóricos, han sido muchos los estudios empíricos realizados sobre el tema en España, aumentado aún más la importancia de los mismos a medida que ha ido aumentando la participación de la mujer en el mercado de trabajo (Arellano et al., 1995). En concreto, para España podemos encontrar numerosos trabajos aplicados sobre este tema, si bien la falta de datos a nivel individual ha condicionado siempre este tipo de estudio. Entre los trabajos existentes para la economía española cabe destacar los trabajos seminales de Moltó (1984), Peinado (1988) y Riboud y Hernandez (1989) así como los más recientes de Ugidos (1994), De la Rica y Ugidos (1995), Hernández (1995), Prieto (1995), García-Crespo (1996), Manero (1999), García (2003) y Simón (2006). En la mayoría de estos trabajos el objetivo fundamental es estimar el grado de discriminación salarial en España, y en algunos como el De la Rica y Ugidos (1995) se analiza en detalle el papel de las diferencias en capital humano sobre dicha discriminación. Algunos autores como Aláez y Ullibarri (2000) han dedicado su estudio al análisis de las diferencias regionales en la discriminación salarial. Otros autores han centrado su estudio en una comunidad autónoma, como es el caso de Aláez y Ullibarri (1999) para la comunidad vasca o Pena-Boquete y Fernández (2006) para la comunidad de Galicia. En cuanto al caso de la comunidad andaluza, hasta donde tenemos constancia, no se ha realizado ningún estudio específico para esta región salvo García-Crespo (2001).

\section{Datos y descripción de las variables}

\subsection{Descripción de la base de datos.}

Los datos utilizados en este trabajo provienen de una muestra del Fichero Técnico de Afiliados y Empresas del Régimen General de la Seguridad Social para el período 1998-2003. La principal ventaja de la utilización de esta base de datos es, aparte de la fiabilidad de la información al tratarse de datos administrativos, que se dispone de un grupo muy amplio de individuos con un período de observación también amplio. Esto cobra especial relevancia si se desea focalizar el estudio en una determinada región española. Las bases de datos utilizadas en los estudios anteriores realizados en nuestro país (Encuesta de Población 
Activa, Panel de Hogares, etc.) no disponen de la muestra suficiente para realizar las estimaciones para las distintas regiones españolas. ${ }^{3}$ Adicionalmente, se dispone de información detallada sobre los salarios de cada individuo, si bien no se dispone del número de horas trabajadas por cada uno de ellos. A priori, el no disponer de esta variable podría suponer una importante carencia dado que normalmente las mujeres trabajan en media menos horas semanales que los hombres. Para mitigar el posible efecto de esta falta de información hemos restringido nuestra muestra de trabajadores a tiempo completo. ${ }^{4}$

También disponemos con esta base de información sobre varias características personales del trabajador: edad, sexo, experiencia laboral previa; características del puesto de trabajo como el grupo de tarifa ó el tipo de contrato y también variables relativas a la empresa donde trabaja, tales como su sector, tamaño (es decir, número de trabajadores), provincia donde está ubicada, etc.

No obstante, esta base de datos presenta algunos inconvenientes. Además de no contar con una medida exacta del nivel de cualificación del trabajador como se comenta más adelante, el principal problema es la observación censurada de los datos salariales. Los salarios observados están sujetos a censura, tanto superior como inferior, debido a la existencia de bases de cotización máxima y mínima establecidas por el Régimen General de la Seguridad Social para cada grupo de cotización. Así, en la muestra completa para el caso de España el número de observaciones con censura superior es de un 8.94\% para el caso de los hombres y de tan sólo un 3.81\% en el caso de las mujeres. Con la censura inferior ocurre lo contrario, en el caso de las mujeres tenemos un $11.94 \%$ de censuras mientras que en el caso de los hombres es del 5.47\%. En la submuestra de Andalucía, esta diferencia se acentúa aún más. Así, en el caso de las mujeres las censuras inferiores y superiores son

\footnotetext{
${ }^{3}$ Como hemos comentado en la introducción para realizar la descomposición de BlinderOaxaca necesitamos estimaciones de ecuaciones de salarios separadas para hombres y mujeres. Esta base de datos posee información suficiente para realizar estas estimaciones, incluso cuando nos restringimos al caso andaluz.

4 No obstante, en base a la Encuesta de Población Activa y considerando una submuestra de trabajadores con similares características a las de nuestro estudio (mismo período muestral, trabajadores a tiempo completo, etc.) vemos que la diferencia en horas trabajadas entre hombres y mujeres está en torno a la hora y media para el caso de España (esto representa alrededor de un 3,75\% de la jornada semanal) y dos horas para el caso andaluz (un 5\% aproximadamente de la jornada semanal). Hay que tener en cuenta, por tanto, que nuestra medida de diferencia salarial puede ser una estimación algo sesgada al alza por no tener en cuenta estas pequeñas diferencias en las horas trabajadas que nuestro análisis no puede considerar por falta de información en los datos.
} 
respectivamente $16.82 \%$ y $2.34 \%$, mientras que en el caso de los hombres apenas existe diferencia entre el número de censuras inferiores y superiores siendo éstas $7.83 \%$ y $6.87 \%$. En cualquier caso, creemos que el mayor tamaño muestral así como la fiabilidad de la información compensan este problema que, además, se puede tratar específicamente en la estimación de nuestro modelo.

De cara a tener una muestra de trabajadores lo más homogénea posible hemos aplicado una serie de filtros a la muestra inicial ${ }^{5}$ eliminando grupos donde el número de observaciones es muy reducido: eliminamos los trabajadores que pertenezcan al sector agrícola así como los empleados en empresas de más de 1,000 trabajadores. Nuestros resultados están basados en la información salarial, en términos de bases de cotización a la Seguridad Social del mes de junio ${ }^{6}$ de cada año. En el caso de individuos que realizan más de un trabajo en ese mes se considera únicamente el de mayor salario, determinado en base a su cotización a la Seguridad Social. Tras tener en cuenta estas consideraciones, la muestra final resultante contiene 352,038 observaciones salariales de las cuales 228,940 son de hombres y 123,098 de mujeres, pertenecientes a 75,591 hombres asalariados y 44,631 mujeres asalariadas de 10,663 empresas diferentes. En la submuestra andaluza el número de observaciones son pertenecientes a 32,322 de hombres y 11,828 de mujeres. $^{7}$

\subsection{Descripción de las variables.}

La principal variable objeto de estudio en este trabajo es la base de cotización (salarios, de aquí en adelante) que se mide en términos mensuales y constantes, en euros de 2003. La definición y construcción del resto de variables utilizadas en nuestro estudio se detalla en el apéndice, si bien merece la pena destacar la construcción de la variable cualificación. La base de datos utilizada no dispone de información sobre la cualificación real, i.e., el nivel educativo del trabajador ${ }^{8}$ si bien sí dispone de su grupo de tarifa (cualificación requerida para el puesto de trabajo desempeñado). Por ello construimos la variable cualificación vía el grupo de tarifa, lo que,

5 El número inicial de hombres en la base de datos es de 127,224 y el de mujeres de 101,595 .

${ }^{6}$ Este mes se suele caracterizar normalmente por pagos de carácter estacional pero no es el caso en los registros de la Seguridad Social pues las pagas extraordinarias están prorrateadas en la base de cotización del trabajador.

7 En la muestra del conjunto de España están incluidas las observaciones para Andalucía.

8 Variable imprescindible en cualquier estudio de salarios dado que su omisión ocasionaría un sesgo por variables omitidas en la estimación. 
por tanto, estará recogiendo el efecto no solo del nivel educativo de cada trabajador sino también el requerido para el puesto de trabajo que ocupa.

En los cuadros 1 a 4, presentamos los estadísticos descriptivos de las variables de mayor interés para la muestra de hombres y mujeres ofreciendo información sobre el salario. Un primer aspecto a destacar es la diferencia entre hombres y mujeres en términos de salarios medios. Se observa que el salario de la mujer, en media, es un $85 \%$ del de un hombre en nuestra muestra. En el caso específico de Andalucía (ver Cuadro 2) la situación es muy parecida (84\%).

Atendiendo a la edad observamos que tanto en la muestra total como en la submuestra andaluza la mitad de la población (tanto masculina como femenina) posee entre 30 y 45 años. La distribución de salarios por grupo de edad es muy parecida para ambos sexos encontrándose las mayores diferencias salariales por sexos en las edades comprendidas entre 46 y 65 años, siendo éstas un 9\% superiores en España que en Andalucía (28\% frente a 19\%).

Con respecto al nivel de cualificación requerido para el puesto, es de esperar que a mayor nivel de cualificación mayor salario pero menos diferencias por género (De la Rica et al., 2005). En la muestra final, la mayoría de la población masculina y femenina (aproximadamente el $80 \%$ ), realizan trabajos que requieren una cualificación media o baja. Las diferencias salariales observadas entre los hombres y mujeres de dichos grupos son aproximadamente del $23 \%$ mientras que para los más cualificados son algo menores, $18 \%$. Si comparamos Andalucía respecto al caso español, las diferencias entre los dos sexos son en la mayoría de los subgrupos ligeramente superiores para el caso andaluz, siendo aproximadamente un 5\% mayor en los grupos de cualificación baja y media.

En cuanto a los sectores productivos o ramas de actividad, la presencia de cada género en cada uno de ellos difiere considerablemente, así como las diferencias salariales dentro de los mismos. Los hombres en nuestra muestra tienen en el sector "mina e industria" su mayor presencia, 28\%, mientras que el sector con una mayor presencia de las mujeres es el "comercio" con un $21 \%$ respecto a la muestra total de mujeres. Son precisamente en estos sectores donde las diferencias salariares son mayores, sólo superadas por "servicios personales" y "finanzas, inmobiliaria e investigación" donde los salarios para los hombres son un $28 \%$ y un $33 \%$, respectivamente, superiores a los de las mujeres.

La mayoría de los trabajadores de nuestra base de datos están contratados en empresas de menos de 250 trabajadores (el 89\% de los hombres y $81 \%$ de las mujeres). Si bien las mayores diferencias salariares 
se encuentran en las empresas de entre 500 y 1000 empleados, llegando a ser de hasta un $46 \%$ entre hombres y mujeres, frente al $10 \%$ registrado en las empresas de 10 a 50 trabajadores. En el caso andaluz destaca la escasa presencia de trabajadores de empresas grandes, de más de 500 trabajadores, principalmente hombres, que representan tan sólo el $2 \%$ de la muestra de hombres.

Atendiendo a la comunidad autónoma es en Asturias y Baleares donde se observan mayores diferencias salariares por género (superiores al 30\%) mientras que son Extremadura y Castilla la Mancha las regiones donde las diferencias son menores (inferiores al 4\%).

Con respecto al tipo de contrato, los porcentajes de individuos con contrato indefinido son mayores para hombres que para mujeres, 68\% y $64.5 \%$ en España y 55,5\% y $61 \%$ en Andalucía. Si bien, los hombres con contrato indefinido ganan un 19\% más que las mujeres con dicho contrato, incrementándose estas diferencias en un $3 \%$ en el caso andaluz.

Finalmente, es interesante señalar el mayor porcentaje de mujeres contratadas por una ETT (16\% frente al 7\% de hombres). No obstante, la diferencia salarial por género originada por esta característica es mínima, en torno al 1.5\%. Al contrario ocurre en el caso andaluz donde el porcentaje aumenta para hombres, siendo muy parecido al de mujeres. Respecto a las diferencias salariales para los trabajadores de ETT, en Andalucía, son cinco puntos porcentuales superiores que en el conjunto de España.

Debido a la censura en los datos, no podemos estudiar con fiabilidad una medida usual para la dispersión salarial como puede ser la varianza. No obstante, podemos mostrar evidencia sobre dicha dispersión en base a los cuantiles de la distribución salarial. El cuadro 3 muestra por ello tres medidas para los salarios: la diferencia entre los percentiles $75 \mathrm{y}$ 25, P75-25, o rango intercuantílico, el cual captura la dispersión alrededor de la mediana, la diferencia entre el percentil 25 y el 50, P50-25, medida que recoge la dispersión en la mitad inferior de la distribución de salarios y la diferencia entre el percentil 75 y el 50, P75-50, medida que recoge la dispersión en la mitad superior de la distribución de salarios. El cuadro 4 recoge la misma información pero centrándose en Andalucía.

Antes de comentar estos estadísticos, conviene observar en los Gráficos 1 a 4 las funciones de densidad empíricas estimadas mediante kernel, para la muestra total y la submuestra andaluza, y para cada género. En comparación con las mujeres, en la distribución para los hombres una gran masa de probabilidad se mueve en la mitad superior de la distribución. Al mismo tiempo, el cuadro 3 muestra que las diferencias entre hombres y mujeres en cuanto a la dispersión en esta parte de la distribución son del 38\%, considerando la medida P75-50, en torno a la 
mediana alcanzan casi el 25\%, y bajo la mediana, 5\%, de acuerdo con la medida P50-25. Es decir, la dispersión salarial para los hombres es mayor que para las mujeres, sobre todo en la parte alta de la distribución salarial, para salarios altos. El fenómeno de los techos de cristal puede estar detrás de este hecho observado (ver De la Rica et al., 2005). Para el caso andaluz lo más notable respecto al caso de España, es que la dispersión es mayor para las mujeres, en torno a la mediana y en la parte baja de la distribución que en el caso de los hombres.

\section{Metodología. Técnicas econométricas}

Hoy día aún persisten desigualdades de ingresos entre trabajadores con igual productividad y en iguales trabajos, solamente porque son de distinto género. Esto se atribuye teóricamente a la discriminación en el mercado laboral. La descomposición de las diferencias salariales medias observadas entre diferencias debidas a diferencias en características, y por tanto no discriminatorias, y las debidas a la existencia de discriminación fue tratada por Blinder (1973) y Oaxaca (1973) y extendida por Oaxaca y Ransom (1994). Esta descomposición identifica dos elementos principales: el primero se asocia a las diferencias en las características observables mientras que el segundo a la diferencia entre los coeficientes estimados y por tanto a la retribución marginal de cada una de estas características. Es este segundo elemento el que podemos calificar como elemento discriminatorio implícito en las diferencias observadas.

Hasta nuestros días han sido muchos los estudios realizados con el objeto de medir la discriminación. En nuestro caso, y dado que nuestra medida salarial está sujeta a doble censura, estudiaremos las diferencias salariales por género usando una generalización del método de descomposición de Oaxaca-Blinder para modelos censurados.

El primer paso de este método consiste en estimar ecuaciones de salarios para hombres y mujeres por separado, con el objeto de conocer cómo retribuye el mercado cada una de las características mencionadas anteriormente cuando controlamos por el resto. Las ecuaciones son las siguientes:

$$
\begin{aligned}
& w_{h i}=X_{h i}^{\prime} \beta_{h}+u_{h i} \\
& w_{m i}=X_{m i}^{\prime} \beta_{m}+u_{m i}
\end{aligned}
$$


Donde el subíndice $h$ hace referencia al hombre y $m$ a la mujer, $w_{i}$ representa el logaritmo del salario mensual del trabajador $i$-ésimo, $X_{i}$ el vector de características individuales que consideramos relevantes para explicar las diferencias salariales, $\beta$ son las tasas de retorno de dichas características, y $u_{i}$ es el correspondiente término de error.

En principio, la técnica más comúnmente usada para describir la posición de un "trabajador medio" en cada grupo de la muestra es la regresión lineal mediante mínimos cuadrados ordinarios (MCO). Si no nos queremos centrar en el individuo medio sino que queremos describir lo que ocurre en las colas de la distribución, esto es, los trabajadores que tienen salarios más bajos y los de salarios más elevados respecto del resto de la población y respecto a cada grupo de características considerado, se deberían utilizar las regresiones cuantílicas (Koenker y Bassett, 1978) que nos proporcionan la posibilidad de dar una descripción más segura de la distribución condicional del salario real, al analizar los cambios en cada una de las colas de la distribución y no solo en la media (Abadie, 1997 y Gardeazabal y Ugidos, 2005). Pero, la distribución de salarios en nuestra muestra sufre un problema, ya explicado anteriormente, de censura superior e inferior. Por tanto, no es aplicable ninguna de estas dos metodologías ya que los coeficientes estudiados no serían consistentes.

Las censuras izquierda y derecha establecidas por las bases de cotización mínima y máxima de la Seguridad Social hacen que la distribución de salarios observados en nuestra muestra sea:

$$
w_{i t}=\left\{\begin{array}{ccc}
w_{1 t} & \text { si } & w_{i t} \leq w_{1 t} \\
w_{i t}^{*} & \text { si } & w_{1 t}<w_{i t}<w_{2 t} \\
w_{2 t} & \text { si } & w_{i t} \geq w_{2 t}
\end{array}\right.
$$

Donde los salarios subyacentes no observados tienen la expresión $w^{*}{ }_{i t}=x_{i t} \beta+u_{i t}$ con $u_{i t} \mid x_{i t} \sim N\left(x_{i t}^{\prime} \beta, \sigma^{2}\right)$ y donde $w_{1 t}, w_{2 t}$ representan las bases de cotización mínima y máxima establecidas por la Seguridad Social en cada período $t$. Como es usual en la literatura plantearemos nuestro modelo en términos logaritmos.

Por tanto, dada esta distribución doblemente censurada, el valor de la esperanza salarial observada tendrá la siguiente expresión:

$$
E\left(w_{i t} \mid x\right)=w_{1 t} \Phi\left(\frac{w_{1 t}-x_{i t}^{\prime} \beta}{\sigma}\right)+w_{2 t}\left(1-\Phi\left(\frac{w_{2 t}-x_{i t}^{\prime} \beta}{\sigma}\right)\right)+x_{i t} \beta\left(\Phi\left(\frac{w_{2 t}-x_{i t}^{\prime} \beta}{\sigma}\right)-\Phi\left(\frac{w_{1 t}-x_{i t}^{\prime} \beta}{\sigma}\right)\right)+
$$




$$
+\sigma\left(\phi\left(\frac{w_{2 t}-x_{i t}^{\prime} \beta}{\sigma}\right)-\phi\left(\frac{w_{1 t}-x_{i t}^{\prime} \beta}{\sigma}\right)\right)
$$

Donde $\Phi$ y $\phi$ son las funciones de distribución y densidad, respectivamente, de la distribución normal.

La estimación de esta expresión se hace por máxima verosimilitud siendo la función de verosimilitud:

$$
\log L=\sum_{i=1}^{n} \ln l_{j}
$$

Donde:

$$
\ln l_{j}=\left\{\begin{array}{ccc}
\ln \Phi\left(\frac{w_{1 t}-x_{i t}^{\prime} \beta}{\sigma}\right) & \text { si } & w_{i t} \leq w_{1 t} \\
\ln \left(1-\Phi\left(\frac{w_{2 t}-x_{i t}^{\prime} \beta}{\sigma}\right)\right) & \text { si } & w_{i t} \geq w_{2 t} \\
-\frac{1}{2}\left(\log \sigma^{2}+\log 2 \pi+\left(\frac{w_{i t}-x_{i t}^{\prime} \beta}{\sigma}\right)^{2}\right) & \text { si } & w_{1 t}<w_{i t}<w_{2 t}
\end{array}\right.
$$

Una modificación adicional que consideraremos en este trabajo es, siguiendo a Arellano et al. (2001), que la varianza de esta distribución salarial puede depender de distintos factores observables. En este caso, supondremos que:

$$
w_{i t}^{*} \mid x_{i t} \sim N\left[\mu\left(x_{i t}\right), \sigma^{2}\left(x_{i t}\right)\right]
$$

Donde $\mu\left(x_{i t}\right) \equiv x_{i t}^{\prime} \beta$ y $\sigma\left(x_{i t}\right) \equiv \exp \left(x_{i t}^{\prime} \gamma\right)$.

Una vez estimadas las ecuaciones salariales para ambos géneros siguiendo esta metodología, la descomposición de Oaxaca-Blinder que empleamos consiste en utilizar los coeficientes estimados anteriormente para descomponer las diferencias salariales medias en tres componentes, dado que en el caso de los modelos censurados la descomposición tradicional cambia (Bauer y Sinning, 2005). En estos modelos de regresión censurada la descomposición de Oaxaca-Blinder tiene dos posibles expresiones, dependiendo de la varianza que se utilice, la de la ecuación de salarios para hombres o la de mujeres:

$$
\Delta_{f}=\left[E_{\beta_{m}, \sigma_{m}}\left(Y_{i m} \mid X_{i m}\right)-E_{\beta_{m}, \sigma_{f}}\left(Y_{i f} \mid X_{i f}\right)\right]+\left[E_{\beta_{m}, \sigma_{f}}\left(Y_{i f} \mid X_{i f}\right)-E_{\beta_{f}, \sigma_{f}}\left(Y_{i f} \mid X_{i f}\right)\right]
$$


$\Delta_{m}=\left[E_{\beta_{m}, \sigma_{m}}\left(Y_{i m} \mid X_{i m}\right)-E_{\beta_{m}, \sigma_{m}}\left(Y_{\text {if }} \mid X_{i f}\right)\right]+\left[E_{\beta_{m}, \sigma_{m}}\left(Y_{\text {if }} \mid X_{\text {if }}\right)-E_{\beta_{f}, \sigma_{f}}\left(Y_{i f} \mid X_{\text {if }}\right)\right] \quad$ [8]

Teniendo en cuenta que cada una de estas esperanzas tiene la siguiente expresión en términos muestrales:

$$
\begin{aligned}
& E_{\widehat{\beta}_{g} \bar{\sigma}_{g}}\left(Y_{i g} \mid X_{i g}\right)=S\left(\widehat{\beta}_{g}, X_{g}, \bar{\sigma}_{g}\right)=N_{g}^{-1} \sum_{i=1}^{N_{g}}\left\{w_{1 t} \Phi\left(\frac{w_{1 t}-x_{i t}^{\prime} \widehat{\beta}_{g}}{\widehat{\sigma}_{g}}\right)+w_{2 t}\left(1-\Phi\left(\frac{w_{2 t}-x_{i t}^{\prime} \widehat{\beta}_{g}}{\widehat{\sigma}_{g}}\right)\right)+\right. \\
& \left.x_{i t} \widehat{\beta}_{g}\left(\Phi\left(\frac{w_{2 t}-x_{i t}^{\prime} \widehat{\beta}_{g}}{\bar{\sigma}_{g}}\right)-\Phi\left(\frac{w_{1 t}-x_{i t}^{\prime} \widehat{\beta}_{g}}{\widehat{\sigma}_{g}}\right)\right)+\bar{\sigma}_{g}\left(\phi\left(\frac{w_{2 t}-x_{i t}^{\prime} \beta_{g}}{\bar{\sigma}_{g}}\right)-\phi\left(\frac{w_{1 t}-x_{i t}^{\prime} \widehat{\beta}_{g}}{\widehat{\sigma}_{g}}\right)\right)\right\}_{[9]}
\end{aligned}
$$

Pero una descomposición que nos proporciona más información es la siguiente:

$$
\begin{aligned}
& \Delta^{*}=\left[S\left(\widehat{\beta}_{m}, X_{i m}, \bar{\sigma}_{m}\right)-S\left(\widehat{\beta}_{m}, X_{i f}, \bar{\sigma}_{m}\right)\right]+\left[S\left(\widehat{\beta}_{m}, X_{i f}, \bar{\sigma}_{m}\right)-S\left(\widehat{\beta}_{f}, X_{i f}, \bar{\sigma}_{m}\right)\right]+ \\
& +\left[S\left(\widehat{\beta}_{f}, X_{i f}, \bar{\sigma}_{m}\right)-S\left(\widehat{\beta}_{f}, X_{i f}, \bar{\sigma}_{f}\right)\right]
\end{aligned}
$$

Donde añadimos un tercer término que controla también las diferencias en la varianza. Esta será la expresión de la descomposición salarial que utilizaremos. El primer término de la derecha de esta última expresión refleja qué parte de las diferencias salariales medias se deben a diferencias en las características observables entre hombres y mujeres, el segundo término refleja la parte de estas diferencias atribuible a la diferencia en los rendimientos medios de dichas características y finalmente el tercer término es la parte de la diferencia salarial debida a la diferente dispersión salarial entre ambos géneros. Este tercer término refleja también discriminación ya que recoge los efectos de las distintas variables explicativas sobre la dispersión salarial estimada para hombres y mujeres.

Hay que tener en cuenta que la medida de la discriminación salarial que calcularemos supondrá que la estructura salarial no discriminatoria es la de los hombres, es decir que en ausencia de discriminación, hombres y mujeres serían retribuidos a los mismos salarios, el de los hombres. Aunque esta es la estructura salarial no discriminatoria más usada, en la literatura aplicada se han propuesto otras posibilidades. Así, Neumark (1988) propone que la estructura no discriminatoria esté entre la femenina y la masculina, en este caso la estructura de toda la población. 
Uno de los problemas que presenta este método e independientemente de lo anteriormente mencionado, como Pena-Boquete y Fernández (2006) señalan, es que sólo se tienen en cuenta el hombre "medio" y la mujer "media" para el cálculo de la discriminación. De este modo estamos suponiendo que la discriminación se distribuye homogéneamente a lo largo de la distribución sin tener en cuenta que puede haber grupos de mujeres más discriminados que otros. De hecho, esta es la motivación para permitir que la varianza dependa de distintas características individuales y de manera diferente para hombres y mujeres, para así dar respuesta en parte a este problema.

Por último, otro problema que puede estar presente en nuestra estimación es el del sesgo de selección muestral. El sesgo de selección consiste en que, al no poder incluir información sobre el salario de quienes no están trabajando, la estimación de los coeficientes posiblemente esté sesgada. Por ejemplo, las personas con salarios de reserva superiores a los de mercado no trabajan, lo que implica que no contemos con información sobre su salario de mercado. Como el tener salarios de reserva superiores a los del mercado no es un fenómeno necesariamente aleatorio, la exclusión de dichas personas hace que la muestra no cumpla con las condiciones de aleatoriedad requeridas por los modelos econométricos. ${ }^{9}$ No obstante, también podemos interpretar las diferencias salariales que nosotros estimamos como la representación conjunta tanto de diferencias puras en remuneración como de diferencias en participación entre ambos grupos poblacionales. Existe una parte de la literatura que considera éstas, las que no eliminan las diferencias en participación, como las que mejor están capturando las diferencias discriminatorias entre hombres y mujeres.

\section{Resultados empíricos}

En esta sección, se presentan los resultados empíricos de las estimaciones salariales de hombres y mujeres, así como la descomposición salarial aplicando el método de Blinder-Oaxaca para la muestra de trabajadores del Régimen General de la Seguridad Social para España, y por la submuestra de Andalucía, en base a sus bases de cotización mensuales del mes de junio de cada año, para el periodo 1998-2003.

\footnotetext{
${ }^{9}$ La estimación del modelo censurado controlando por participación, es algo que sin duda haría más eficientes nuestros resultados de estimación, al igual que controlar la heterogeneidad no observable mediante técnicas de panel. Estas dos modificaciones están en nuestra agenda futura de investigación.
} 


\subsection{Estimaciones salariales}

Los resultados básicos de estas estimaciones se pueden encontrar en los cuadros 5 y 6 . El cuadro 5 contiene las estimaciones realizadas en la muestra española con un modelo censurado y con estimación de la varianza, una para cada sexo. En todas las regresiones la variable dependiente es el logaritmo del salario real mensual. En todas ellas también se ha controlado por efectos fijos temporales, y además, en el caso de la muestra española se ha controlado por efectos regionales.

Dichas regresiones son estimadas considerando como grupo de referencia para la muestra total española, un trabajador de una empresa dedicada al comercio de menos de 10 empleados, nativo, ${ }^{10}$ con baja cualificación, de menos de 6 meses de antigüedad en el puesto de trabajo, no contratado por una ETT, sin trabajo fijo y residente en la comunidad de Madrid. En el caso de la submuestra andaluza, el individuo de referencia posee las mismas características excepto, obviamente, la referida a su región de residencia.

Centrándonos en las diferencias estimadas entre hombres y mujeres, comenzando por los rendimientos de las variables relativas a las características individuales, destacan los siguientes resultados. Con respecto a la cualificación requerida para el puesto se observa que tanto para hombres como para mujeres, y tanto para la muestra española como para la submuestra andaluza, la cualificación aumenta el salario considerablemente respecto al grupo de referencia (individuos que ocupan empleos que requieren baja cualificación). Concretamente, en el caso de España, el retorno a puestos con la más alta cualificación se sitúa entorno al 70\% respecto al del grupo de referencia en el caso de los hombres, y alrededor de un 65\% en el caso de las mujeres. En el caso de Andalucía estas cifras se aproximan al $60 \%$ para ambos géneros. Esta menor remuneración relativa de los puestos de cualificación altos en Andalucía podría deberse a la menor presencia en esta región de sectores en los que se demandan empleados con altos niveles de cualificación. Asimismo, una mayor influencia de la negociación colectiva, en términos de compresión salarial, también podría estar detrás de este resultado.

En lo relativo al tamaño de la empresa, se observa que los rendimientos son crecientes en el tamaño tanto para Andalucía como para España, siendo los salarios notablemente mayores en las empresas de

\footnotetext{
10 Hemos repetido nuestro análisis estimando por separado para nativos e inmigrantes y las diferencias salariales entre hombres y mujeres, así como la descomposición presentada más adelante, se mantienen prácticamente inalterados. Los resultados se encuentran a disposición del lector interesado.
} 
mayor tamaño. Si bien, en el caso andaluz, en la mayoría de los grupos y para ambos géneros se observa un notable incremento en los retornos con el tamaño de la empresa respecto al caso Español. La distancia, por tanto, en la remuneración en microempresas (menos de 10 trabajadores) y grandes empresas es mucho mayor en Andalucía, lo cual podría deberse al menor grado de competencia que pueda estar teniendo el sector de pequeñas empresas en esta región, lo que les puede ayudar a fijar salarios mucho menores a los que fijan las empresas de mayor tamaño.

Respecto a la antigüedad en el trabajo actual, esta variable afecta de forma muy parecida a hombres y mujeres con un comportamiento similar en Andalucía y España, siendo entre un 15 y un 18\% mayor la remuneración cuando la antigüedad es de más de 6 años frente a los que llevan menos de seis meses en la empresa.

La variable asociada con el tipo de contrato de duración indefinida ${ }^{11}$ tiene signo positivo tanto para hombres como para mujeres, lo que implica que dicho tipo de contrato aumenta las ganancias en relación con el grupo de referencia, que son individuos con contrato temporal. Sin embargo, la magnitud es mayor para las mujeres que para los hombres, siendo esta diferencia aún mayor en el caso andaluz. Así, cuando controlamos por una serie de características, el retorno marginal ${ }^{12}$ a tener un contrato indefinido es, respecto al grupo de referencia, del 13\% en los hombres y del $19 \%$ en las mujeres, para el caso español, frente al $15 \%$ y $20 \%$ entre hombres y mujeres andaluces respectivamente. Por tanto, las mujeres obtienen un mayor retorno a ser contratadas bajo un contrato indefinido, sobre todo si lo hacen en empresas grandes o en el Sector Público, y en Andalucía. En el conjunto del Estado Español, este mayor retorno parece provenir de la mayor proporción de mujeres en puestos de cualificación media o superior.

El coeficiente asociado con la variable trabajar en una ETT es positivo para las mujeres y negativo para los hombres. Más

\footnotetext{
11 Tanto la variable contrato indefinido como ETT han sido usadas en la especificación final acompañadas de varios términos de interacción con algunas características del puesto de trabajo. En principio hemos interactuado esta variable con todas las categorías de cualificación pero los resultados no eran significativos más que para las interacciones mostradas en el cuadro 5. Interactuar las variables ETT y fijo con algunas otras tiene como objeto ver el efecto concreto de poseer un contrato indefinido o bien de ser contratado a través de una ETT sobre estos subgrupos muestrales.

12 Nótese que al tratarse de una estimación teniendo en cuenta la censura los coeficientes de la regresión no se puede interpretar como los efectos marginales, tal y como haríamos e.g. en el caso de estimaciones por MCO. En las variables comentadas en este apartado los efectos marginales comentados son calculados haciendo uso de la expresión para la media mostrada en [9].
} 
concretamente, el retorno marginal a ser contratado a través de una ETT es de $-2 \%$ y $-3 \%$ en hombres españoles y andaluces respectivamente, mientras que en el caso de las mujeres existen mayores diferencias entre las que han sido contratadas por una ETT y trabajan en Andalucía o en el conjunto de España (1\% frente a un 12\%). El por qué esta variable puede afectar de manera tan distinta a hombres y mujeres podría venir explicado porque los tipos de trabajos a los que acceden hombres y mujeres a través de una ETT son muy diferentes. Los trabajos a los acceden los hombres contratados a través de una ETT son de salarios más bajos. En el caso de las mujeres parece ocurrir lo contrario.

Los hombres inmigrantes ganan un salario ligeramente inferior a los nativos mientras que en el caso de las mujeres es al contrario. Aunque a nivel descriptivo los salarios de las nativas son superiores a los de las inmigrantes, el efecto es el contrario en el ejercicio econométrico donde comparamos nativas e inmigrantes con iguales características observadas: las inmigrantes parecen recibir una remuneración algo superior a las nativas lo que puede estar debiéndose a que otras características no observadas en nuestra base de datos, como por ejemplo el nivel educativo, estén influyendo en el resultado.

Atendiendo a la desviación típica estimada se observa que la dispersión salarial disminuye con la edad en el caso de los hombres, produciéndose el resultado contrario en el caso de las mujeres, tanto en la muestra total como en la submuestra andaluza. Con respecto al nivel de cualificación requerido para el puesto, la dispersión salarial aumenta con el mismo para ambos géneros, si bien no en la misma magnitud. En el caso de los hombres la desviación típica ${ }^{13}$ es un $53 \%$ mayor para los empleados en puestos de cualificación alta que para los que se encuentran en el grupo de referencia, situándose esta diferencia en las mujeres en un $31 \%$. Por otra parte, se observa que, en general, estos retornos son mayores en la submuestra andaluza. El efecto de la antigüedad en el empleo sobre la dispersión es el inverso, es decir, al aumentar la antigüedad en el empleo la dispersión salarial disminuye, y lo hace en menor medida en las mujeres que en los hombres. El hecho de tener un contrato indefinido hace que la dispersión salarial sea menor que entre los que tienen un contrato temporal, siendo este efecto mayor en las mujeres. La dispersión salarial estimada en los trabajadores contratados a través de una ETT tiene el mismo efecto para ambos géneros: la dispersión es mayor para los contratados en una ETT que en los del grupo de referencia, cuando

\footnotetext{
${ }^{13}$ Cuando hablamos en este caso de dispersión nos estamos refiriendo a los coeficientes
} estimados para el argumento de la función exponencial de la desviación típica. 
controlamos por una serie de características, y es, a su vez, mayor en mujeres que en hombres.

\subsection{Resultados de la descomposición salarial.}

Los resultados de la descomposición salarial en base a las ecuaciones estimadas se presentan en el cuadro 7. Recordemos que podemos descomponer la diferencia salarial media observada entre hombres y mujeres en diferencias en características y en rendimientos, y éstos últimos tanto para la media como para la varianza. Además, cada uno de estos componentes contiene variables que tienden tanto a aumentar como a disminuir la diferencia salarial. La combinación de estos efectos positivos y negativos da como resultado la diferencia salarial observada.

Tanto en el modelo de regresión lineal que sabemos incorrecto pues no controla adecuadamente el problema de la censura, como en el modelo censurado estándar, esto es, con varianza constante, se obtiene que, de los 17 puntos porcentuales de diferencias observadas entre hombres y mujeres, un $77 \%$ es debido a las diferencias en el retorno a la media, mientras que el $23 \%$ restante se debe a diferencias en las características observables. Por tanto, las diferencias que se pueden explicar por diferencias en los retornos, es decir por discriminación, son de 13 puntos porcentuales con respecto al salario medio de los hombres. Cuando usamos el modelo en el que estimamos la varianza, en la descomposición de Oaxaca-Blinder, obtenemos que el componente de discriminación aumenta hasta alcanzar un $83 \%$. Por otra parte, obtenemos que las diferencias en la varianza contribuyen negativamente a explicar las diferencias entre hombres y mujeres. En otras palabras, si las características medias de los hombres fueran las mismas que las de las mujeres, la diferencia salarial entre hombres y mujeres sería de 14 puntos porcentuales, es decir, menor a la observada (17 puntos porcentuales). En lo relativo a los retornos a la varianza para las distintas variables explicativas, se obtiene que son las mujeres las que tienen una mayor varianza salarial, lo que contribuye a rebajar las diferencias salariales observadas. Si los retornos a la varianza fuesen iguales entre hombres y mujeres, observaríamos unas diferencias salariales que serían un 2.52\% superiores.

La interpretación de los resultados para el resto de modelos y las distintas submuestras es análoga. Varios aspectos nos parecen interesantes destacar. En primer lugar y con respecto a la submuestra de Andalucía, las diferencias en el retorno a la medias son un 5\% menores, aproximadamente, respecto al caso de España en los modelos MCO y 
censurado estándar, siendo esta diferencia aún mayor en el modelo censurado con estimación de la varianza. En este último modelo, la parte que nos mide la discriminación baja un 11\% respecto a lo que mide el caso de España. Por tanto, obtenemos que, neto de diferencias en las características observables, la discriminación salarial media en Andalucía es de 12.6 puntos porcentuales frente a casi 14 puntos en el conjunto del Estado español.

Por último, también nos interesa estudiar si esta descomposición es muy distinta para puestos de trabajo que requieren alta o baja cualificación. Para ello, hemos estimado nuestro modelo con dos submuestras (de la muestra total, i.e., incluyendo Andalucía): una que recoge trabajadores en puestos de alta cualificación y otra con los de baja cualificación, y los resultados obtenidos nos permiten obtener la descomposición mostrada en los dos últimos paneles del cuadro 7. En el caso de la submuestra de personas con cualificación alta, la descomposición estudiada nos dice que de los 16 puntos porcentuales observados en diferencias salariales, tan sólo un $27 \%$ se debe a las diferencias en los retornos a la media. Lo que se ajusta a la idea de que a medida que sube el nivel de cualificación la discriminación es menor. En el caso de las personas en puestos de baja cualificación ocurre exactamente lo contrario, la parte de las diferencias observadas correspondiente a las diferencias en los retornos a la media es de casi el 84\% de las diferencias observadas. Por tanto, podemos concluir que las diferencias salariales debidas a discriminación son de solo 4.3 puntos porcentuales para cualificados y de 15.6 puntos porcentuales para no cualificados.

\section{Conclusiones}

El objetivo de este trabajo es analizar los factores explicativos de las diferencias salariales por género en España y en Andalucía en particular, con la finalidad de obtener una medida ajustada de discriminación salarial por género para ambos ámbitos territoriales. Para ello se han estimado ecuaciones de salarios (suponiendo que la varianza no es constante sino dependiente de distintas características individuales) teniendo en cuenta que los datos de los que disponíamos están censurados por las bases de cotización máxima y mínima establecidas por el Régimen General de la Seguridad Social. Los datos utilizados en este estudio provienen de una muestra del Fichero Técnico de Afiliados y Empresas del Régimen General de la Seguridad Social para el período 1998-2003. Por tanto, una importante aportación de este estudio es la consideración de una medida muy ajustada del salario para una muestra muy amplia de 
trabajadores, más de 75,000 hombres y más de 44,000 mujeres asalariadas en 10,663 empresas diferentes. Además, se trata de controlar las diferencias observadas entre hombres y mujeres no solo en su retribución media sino también en una medida de dispersión salarial, en su varianza salarial.

Una vez estimadas nuestras ecuaciones de salarios, procedemos a la típica descomposición de las diferencias mediante una generalización para modelos censurados de la Descomposición de Oaxaca-Blinder. Este método aplicado a los datos comentados nos indica que las diferencias salariales debidas a discriminación entre hombres y mujeres son del $14.39 \%$ para el conjunto de España y del $12.84 \%$ en la comunidad autónoma de Andalucía. Esto es, a pesar de que las diferencias observadas en media (ver Cuadros 1 y 2) son algo mayores en Andalucía, 18.06\%, que en el conjunto del Estado Español, 17.87\%, una vez que controlamos adecuadamente por los problemas de censura en los datos y por las diferencias no solo en las características individuales entre hombres y mujeres sino también en las diferencias en sus varianzas salariales, emerge un problema de discriminación importante pero de una entidad algo menor a la tradicionalmente citada, las diferencias brutas entre salarios observados.

Otro resultado interesante de nuestro trabajo aparece cuando dividimos la muestra completa para España entre trabajadores en puestos de alta y de baja cualificación. En concreto, se observa una clara evidencia de que la discriminación salarial por género está presente básicamente en estos últimos, en los puestos de trabajo de baja cualificación donde, controlando por las diferencias en características individuales y en retornos a la varianza, la discriminación por género es de 15.6 puntos porcentuales en este grupo mientras que es de solo 4.3 puntos porcentuales en los puestos de alta cualificación. Esto nos indica que si, como se demuestra en Dolado et al. (2004), la mujer también está sujeta a discriminación ocupacional, la evidencia de discriminación por género puede ser aun más intensa que la encontrada en este trabajo.

\section{Bibliografía}

Abadie, A. (1997): "Change in the Spanish Labor Income structure during the 1980's: A quantile regression approach," Investigaciones Económicas, 21 , pp. $253-272$.

Aigner, D.J. and Cain G.G. (1977): "Statistical theories of discrimination in labour markets", Industrial and Labour Relations Review, 30 (2), pp. $175-187$. 
Aláez, R., Ullibarri, M. (1999): "Discriminación salarial por sexo en la Comunidad Autónoma Vasca: un análisis del sector privado y sus diferencias con España”, Ekonomiaz, Revista Vasca de Economía, 45, pp. 284-303.

Aláez, R., Ullibarri, M. (2000): "Discriminación salarial por sexo: un análisis del sector privado y sus diferencias regionales en España", Información Comercial Española, 789, pp. 117-138.

Arellano M., Bentolila, S., Bover, O. (2001): “The Distribution of Earnings In Spain During The 1980s: The effects of Skill, Unemployment, and Union Power". CEPR. Discussion Paper Series. No. 2770.

Arellano, M., Bover, O. (1995): "Female Labour Force Participation in the 1980's: The Case of Spain”, Investigaciones Económicas, 29, pp. 171-194.

Bauer, TK., Sinning, M. (2005): "Blinder-Oaxaca Decomposition for Tobit Models". Discussion Paper Series. Institute for the Study of Labor (IZA DP. 1795).

Becker, G. S. (1971): The Economics of Discrimination, Chicago, University of Chicago Press (Edición original, 1957).

Becker, G. S. (1975): Human Capital. A theoretical and Empirical Analysis with Special Reference to Education, Columbia University Press, New York.

Bergmann, B. (1986): The Economic Emergence of Women, Basic Books, New York.

Blinder, A S. (1973): "Wage Discrimination: Reduced Form and Structural Variables", Journal of Human Resources, 8, pp. 436-455.

Brown, R. S. (1980): "Incorporating Occupational Attainment in Studies of Male-Female Earnings Differentials", Journal of Human Resources, 15, pp. $3-28$.

De la Rica, S., Dolado, J.J., Llorent, V. (2005): "Ceiling and Floors: Gender Wage by Education in Spain.” IZA Discussion Paper 1483.

De la Rica, S., Ugidos, A. (1995): “¿Son las diferencias en capital humano determinantes de las diferencias salariales observadas entre hombres y mujeres?", Investigaciones Económicas, 19(3), pp. 395 - 414.

Dolado, J.J., Felgueroso, F., y Jimeno, J.F. (2004): "Where do Women Work?: Analyzing Patterns in Occupational Segregation By Gender", Annales d'Economie et de Statistique, 71-72, pp. 293-315. 
García, J.R. (2003): "La desigualdad Salarial en España. Efectos de un Diseño Muestral Complejo”. FEDEA Documento de Trabajo 2003-26.

García-Crespo, M.D. (1996): "Movilidad Profesional y Discriminación Salarial en el Mercado de Trabajo Español”. Tesis Doctoral. Universidad de Málaga.

García-Crespo, M.D. (2001): "Discriminación Salarial por Sexo en Andalucía”, mimeo, Universidad de Málaga.

García, J., Hernández, P.J., López-Nicolás, A. (2001), "How Wide is the Gap? An investigation of Gender Wage Differences using Quantile Regression”, Empirical Economics, 29, pp. 149 - 168.

Gardeazabal, J., Ugidos, A. (2005): "Gender wages discrimination at quantiles". Journal of Population Economics, 18(1), pp.165-179.

Hernández, PJ. (1995): "Análisis Empírico de la Discriminación Salarial de la Mujer en España”. Investigaciones Económicas, 19(2), pp. 195 - 215.

Koenker, R., Bassett, G. (1978): "Regression Quantiles", Econometrica, Vol. 46, No. 1.

Manero, M. (1999): "La discriminación salarial en el mercado de trabajo español”, Tesina CEMFI no. 9906.

Moltó, M.L. (1984): "Estudio empírico de la discriminación de la mujer en el mercado de trabajo académico”, Estadística Española, 102, pp. 105-128.

Neumark, D. (1988): "Employers Discriminatory behaviour and the estimation of wage discrimination", Journal of Human Resources, 23, pp. 279-295.

Oaxaca, R. (1973): "Male-female wage differentials in urban labour markets", International Economic Review, 14(3), pp. 693-709.

Oaxaca, R., Ransom, M. (1994): "On Discrimination and the Decomposition of Wage Differentials", Journal of Econometrics, 61, pp. 521.

Peinado, A. (1988): La discriminación de la mujer en el Mercado de trabajo español: una aproximación empírica a la discriminación salarial, Ministerio de Trabajo y Seguridad Social, Colección Informes: Serie Empleo.

Pena-Boquete, Y., Fernández, M. (2006): "A Comparative Analysis of the Evolution of Gender Wage Discrimination: Spain vs. Galicia”. Documento de Trabajo, Universidad de York. 
Prieto, J. (1995): "Discriminación Salarial de la Mujer y Movilidad Laboral". Tesis doctoral, Universidad de Oviedo.

Riboud, M. y F. Hernández (1989): Un análisis de la discriminación de las mujeres en España, Ministerio de Asuntos Sociales, Instituto de la Mujer, Madrid.

Simón, H. (2006): "Diferencias Salariales entre hombres y mujeres en España: Una comparación Internacional con datos emparejados EmpresaTrabajador". Investigaciones Económicas, 30(1), pp. 55- 87.

Ugidos, A. (1994): "Gender Wage Differentials and Sample Selection: Evidence from Spain”. Documento de Trabajo 94.01. Departamento de Teoría Económica, Economía Aplicada II y III e Instituto de Economía Pública, Universidad del País Vasco. 
Cuadro 1. Salarios mensuales por género: media y desviación típica (España)

\begin{tabular}{|c|c|c|c|c|c|c|c|c|c|}
\hline & \multicolumn{3}{|c|}{ HOMBRES } & \multicolumn{3}{|c|}{ MUJERES } & \multirow{2}{*}{$\begin{array}{l}\text { Diferencia } \\
\text { en la media }\end{array}$} & \multirow{2}{*}{$\begin{array}{c}\text { Diferencia } \\
\text { en la } \\
\text { desviación }\end{array}$} \\
\hline & & Observ. & Media & $\begin{array}{c}\text { Desviación } \\
\text { típica }\end{array}$ & Observ. & Media & $\begin{array}{c}\text { Desviación } \\
\text { típica }\end{array}$ & & \\
\hline \multicolumn{2}{|c|}{ muestra completa } & $100 \%$ & $1.372,8$ & 599,35 & $100 \%$ & $1.164,7$ & 541,79 & $17,87 \%$ & $10,62 \%$ \\
\hline \multirow{3}{*}{ edad } & $16-29$ & $25,72 \%$ & $1.088,7$ & 434,36 & $32,47 \%$ & 996,7 & 414,17 & $9,23 \%$ & $4,88 \%$ \\
\hline & $30-45$ & $48,27 \%$ & $1.426,3$ & 605,14 & $48,44 \%$ & $1.256,5$ & 579,18 & $13,51 \%$ & $4,48 \%$ \\
\hline & $46-65$ & $26,01 \%$ & $1.554,4$ & 629,96 & $19,09 \%$ & $1.217,3$ & 567,87 & $27,68 \%$ & $10,93 \%$ \\
\hline \multicolumn{2}{|c|}{ inmigrante } & $2,06 \%$ & $1.027,8$ & 394,38 & $1,30 \%$ & 904,4 & 380,67 & $13,64 \%$ & $3,60 \%$ \\
\hline \multirow{5}{*}{ cualificación } & alta & $8,94 \%$ & $2.084,9$ & 657,09 & $13,85 \%$ & $1.768,7$ & 594,38 & $17,88 \%$ & $10,55 \%$ \\
\hline & media - alta & $9,25 \%$ & $1.945,9$ & 646,96 & $5,85 \%$ & $1.678,8$ & 677,31 & $15,91 \%$ & $-4,48 \%$ \\
\hline & media & $12,52 \%$ & $1.440,3$ & 572,50 & $18,16 \%$ & $1.253,4$ & 503,24 & $14,91 \%$ & $13,76 \%$ \\
\hline & media - baja & $40,10 \%$ & $1.282,0$ & 478,87 & $28,38 \%$ & $1.042,6$ & 389,62 & $22,96 \%$ & $22,91 \%$ \\
\hline & baja & $29,19 \%$ & $1.068,7$ & 380,82 & $33,77 \%$ & 882,9 & 299,54 & $21,05 \%$ & $27,14 \%$ \\
\hline \multirow{10}{*}{ sectores } & comercio & $18,34 \%$ & $1.329,0$ & 585,26 & $20,81 \%$ & $1.042,3$ & 475,16 & $27,51 \%$ & $23,17 \%$ \\
\hline & mina e industria & $27,89 \%$ & $1.481,1$ & 589,70 & $17,79 \%$ & $1.165,0$ & 494,44 & $27,13 \%$ & $19,27 \%$ \\
\hline & construcción & $20,91 \%$ & $1.178,2$ & 463,69 & $2,26 \%$ & $1.207,2$ & 505,41 & $-2,40 \%$ & $-8,25 \%$ \\
\hline & hosteleria & $4,40 \%$ & $1.089,3$ & 440,22 & $7,15 \%$ & 950,1 & 330,22 & $14,65 \%$ & $33,31 \%$ \\
\hline & $\begin{array}{l}\text { transporte y } \\
\text { telecom. }\end{array}$ & $7,25 \%$ & $1.471,5$ & 645,90 & $2,76 \%$ & $1.380,1$ & 620,67 & $6,62 \%$ & $4,06 \%$ \\
\hline & $\begin{array}{l}\text { finanzas, } \\
\text { inmobiliaria, } \\
\text { investigación }\end{array}$ & $10,75 \%$ & $1.559,8$ & 744,56 & $19,23 \%$ & $1.170,5$ & 601,03 & $33,26 \%$ & $23,88 \%$ \\
\hline & sector público & $2,09 \%$ & $1.376,0$ & 476,16 & $3,96 \%$ & $1.392,2$ & 535,66 & $-1,16 \%$ & $-11,11 \%$ \\
\hline & educación & $2,92 \%$ & $1.563,7$ & 589,38 & $8,03 \%$ & $1.530,0$ & 583,66 & $2,20 \%$ & $0,98 \%$ \\
\hline & sanidad & $1,77 \%$ & $1.505,5$ & 618,52 & $11,57 \%$ & $1.218,4$ & 518,45 & $23,56 \%$ & $19,30 \%$ \\
\hline & servicios & $3,68 \%$ & $1.256,3$ & 595,12 & $6,44 \%$ & 980,9 & 507,45 & $28,07 \%$ & $17,28 \%$ \\
\hline \multirow{6}{*}{ tamaño empresa } & $1-10$ & $24,45 \%$ & $1.132,9$ & 484,57 & $24,47 \%$ & 976,2 & 437,25 & $16,05 \%$ & $10,82 \%$ \\
\hline & $11-50$ & $34,83 \%$ & $1.287,5$ & 539,45 & $27,86 \%$ & $1.169,9$ & 533,27 & $10,05 \%$ & $1,16 \%$ \\
\hline & $51-100$ & $13,24 \%$ & $1.492,8$ & 611,34 & $12,83 \%$ & $1.247,8$ & 542,55 & $19,63 \%$ & $12,68 \%$ \\
\hline & ${ }^{\mathrm{a}} 101-250$ & $16,46 \%$ & $1.526,3$ & 615,27 & $15,32 \%$ & $1.251,1$ & 591,62 & $22,00 \%$ & $4,00 \%$ \\
\hline & $251-500$ & $5,50 \%$ & $1.789,7$ & 682,22 & $10,93 \%$ & $1.293,3$ & 592,31 & $38,38 \%$ & $15,18 \%$ \\
\hline & $500-1000$ & $5,52 \%$ & $1.811,2$ & 609,28 & $8,60 \%$ & $1.242,3$ & 536,71 & $45,80 \%$ & $13,52 \%$ \\
\hline \multirow{10}{*}{$\begin{array}{c}\text { antigüedad en } \\
\text { el puesto } \\
\text { (contrato } \\
\text { actual) }\end{array}$} & $1-6$ meses & $24,30 \%$ & $1.105,0$ & 505,69 & $26,64 \%$ & 964,7 & 437,44 & $14,54 \%$ & $15,60 \%$ \\
\hline & $6-12$ meses & $11,59 \%$ & $1.228,8$ & 529,05 & $13,75 \%$ & $1.068,2$ & 497,38 & $15,03 \%$ & $6,37 \%$ \\
\hline & $12-18$ meses & $10,17 \%$ & $1.375,6$ & 586,28 & $9,27 \%$ & $1.159,7$ & 515,90 & $18,61 \%$ & $13,64 \%$ \\
\hline & $18-24$ meses & $5,87 \%$ & $1.361,4$ & 595,37 & $6,22 \%$ & $1.184,9$ & 554,07 & $14,90 \%$ & $7,45 \%$ \\
\hline & $24-30$ meses & $5,54 \%$ & $1.399,1$ & 594,61 & $5,44 \%$ & $1.179,1$ & 523,70 & $18,66 \%$ & $13,54 \%$ \\
\hline & $30-36$ meses & $4,12 \%$ & $1.415,3$ & 602,24 & $4,28 \%$ & $1.209,4$ & 557,33 & $17,03 \%$ & $8,06 \%$ \\
\hline & 3 - 4 años & $6,60 \%$ & $1.469,6$ & 606,39 & $6,94 \%$ & $1.218,6$ & 542,95 & $20,60 \%$ & $11,68 \%$ \\
\hline & $4-5$ años & $5,09 \%$ & $1.515,5$ & 607,18 & $4,98 \%$ & $1.270,0$ & 556,40 & $19,33 \%$ & $9,13 \%$ \\
\hline & $5-6$ años & $4,06 \%$ & $1.567,5$ & 615,99 & $3,64 \%$ & $1.343,1$ & 578,98 & $16,71 \%$ & $6,39 \%$ \\
\hline & más 6 años & $22,67 \%$ & $1.625,8$ & 587,70 & $18,83 \%$ & $1.417,1$ & 576,14 & $14,73 \%$ & $2,01 \%$ \\
\hline \multirow{17}{*}{ ccaa } & Andalucía & $14,12 \%$ & $1.257,5$ & 566,07 & $9,61 \%$ & $1.065,1$ & 504,06 & $18,06 \%$ & $12,30 \%$ \\
\hline & Aragón & $3,03 \%$ & $1.411,2$ & 538,71 & $1,82 \%$ & $1.103,1$ & 465,69 & $27,94 \%$ & $15,68 \%$ \\
\hline & Asturias & $4,03 \%$ & $1.784,1$ & 615,73 & $2,29 \%$ & $1.370,3$ & 628,75 & $30,20 \%$ & $-2,07 \%$ \\
\hline & Baleares & $2,64 \%$ & $1.497,0$ & 632,66 & $2,26 \%$ & $1.149,9$ & 479,92 & $30,18 \%$ & $31,82 \%$ \\
\hline & Canarias & $5,57 \%$ & $1.151,9$ & 522,94 & $6,37 \%$ & $1.044,7$ & 539,86 & $10,26 \%$ & $-3,13 \%$ \\
\hline & Cantabria & $1,42 \%$ & $1.293,0$ & 503,07 & $0,93 \%$ & $1.088,7$ & 509,15 & $18,77 \%$ & $-1,19 \%$ \\
\hline & Castilla-León & $3,08 \%$ & $1.099,4$ & 395,07 & $3,02 \%$ & $1.016,1$ & 433,42 & $8,20 \%$ & $-8,85 \%$ \\
\hline & Castilla-LM & $5,29 \%$ & $1.192,6$ & 436,11 & $6,14 \%$ & $1.147,9$ & 473,11 & $3,89 \%$ & $-7,82 \%$ \\
\hline & Cataluña & $18,41 \%$ & $1.543,0$ & 630,15 & $25,13 \%$ & $1.243,9$ & 561,95 & $24,05 \%$ & $12,14 \%$ \\
\hline & Valencia & $9,52 \%$ & $1.193,1$ & 497,97 & $9,70 \%$ & $1.005,0$ & 420,79 & $18,72 \%$ & $18,34 \%$ \\
\hline & Extremadura & $1,39 \%$ & $1.081,7$ & 457,76 & $1,10 \%$ & $1.069,4$ & 483,02 & $1,15 \%$ & $-5,23 \%$ \\
\hline & Galicia & $7,29 \%$ & $1.221,5$ & 522,17 & $8,44 \%$ & $1.089,2$ & 514,12 & $12,14 \%$ & $1,57 \%$ \\
\hline & Madrid & $15,69 \%$ & $1.487,2$ & 648,81 & $14,15 \%$ & $1.297,4$ & 604,19 & $14,62 \%$ & $7,39 \%$ \\
\hline & Murcia & $2,38 \%$ & $1.246,2$ & 536,27 & $1,84 \%$ & $1.005,3$ & 470,28 & $23,96 \%$ & $14,03 \%$ \\
\hline & Navarra & $1,25 \%$ & $1.654,6$ & 598,27 & $1,93 \%$ & $1.292,9$ & 549,31 & $27,97 \%$ & $8,91 \%$ \\
\hline & P. Vasco & $4,00 \%$ & $1.580,1$ & 598,79 & $4,66 \%$ & $1.259,4$ & 553,94 & $25,46 \%$ & $8,10 \%$ \\
\hline & Rioja & $0,90 \%$ & $1.451,5$ & 478,10 & $0,61 \%$ & $1.232,7$ & 460,31 & $17,75 \%$ & $3,86 \%$ \\
\hline \multirow{2}{*}{\multicolumn{2}{|c|}{$\begin{array}{l}\text { trabajando en una ETT } \\
\text { contrato indefinido }\end{array}$}} & $7,24 \%$ & $1.531,2$ & 606,49 & $16,16 \%$ & $1.509,0$ & 578,64 & $1,47 \%$ & $4,81 \%$ \\
\hline & & $67,72 \%$ & $1.513,3$ & 612,89 & $64,47 \%$ & $1.274,4$ & 549,71 & $18,75 \%$ & $11,49 \%$ \\
\hline
\end{tabular}

Nota: La muestra de estimación contiene 228,940 salarios para hombres y 123,098 para mujeres. En total, cuenta con información sobre 10,663 empresas y 352,038 trabajadores.

Fuente: Muestra del Fichero de Afiliados y Empresas del Régimen General de la Seguridad Social. Elaboración Propia. 
Cuadro 2. Salarios mensuales por género: media y desviación típica (Andalucía)

\begin{tabular}{|c|c|c|c|c|c|c|c|c|c|}
\hline & & \multicolumn{3}{|c|}{ HOMBRES } & \multicolumn{3}{|c|}{ MUJERES } & \multirow[b]{2}{*}{$\begin{array}{l}\text { Diferencia } \\
\text { en la media }\end{array}$} & \multirow{2}{*}{$\begin{array}{c}\text { Diferencia } \\
\text { en la } \\
\text { desviación }\end{array}$} \\
\hline & & Observ. & Media & $\begin{array}{c}\text { Desviación } \\
\text { típica }\end{array}$ & Observ. & Media & $\begin{array}{c}\text { Desviación } \\
\text { típica }\end{array}$ & & \\
\hline \multicolumn{2}{|c|}{ muestra completa } & $100 \%$ & $1.257,5$ & 566,07 & $100 \%$ & $1.065,1$ & 504,06 & $18,06 \%$ & $12,30 \%$ \\
\hline \multirow{3}{*}{ edad } & $16-29$ & $28,26 \%$ & $1.010,6$ & 397,96 & $35,56 \%$ & 914,2 & 381,25 & $10,55 \%$ & $4,38 \%$ \\
\hline & $30-45$ & $50,05 \%$ & $1.320,1$ & 581,78 & $46,68 \%$ & $1.127,2$ & 522,09 & $17,11 \%$ & $11,43 \%$ \\
\hline & $46-65$ & $21,70 \%$ & $1.434,8$ & 608,42 & $17,76 \%$ & $1.204,1$ & 590,14 & $19,15 \%$ & $3,10 \%$ \\
\hline \multicolumn{2}{|c|}{ inmigrante } & $0,62 \%$ & 914,1 & 407,48 & $0,41 \%$ & 873,4 & 252,13 & $4,66 \%$ & $61,62 \%$ \\
\hline \multirow{5}{*}{ cualificación } & alta & $6,48 \%$ & $1.983,2$ & 654,75 & $11,80 \%$ & $1.674,3$ & 545,08 & $18,45 \%$ & $20,12 \%$ \\
\hline & media - alta & $8,07 \%$ & $1.986,5$ & 663,02 & $4,35 \%$ & $1.603,1$ & 694,16 & $23,92 \%$ & $-4,49 \%$ \\
\hline & media & $12,32 \%$ & $1.422,8$ & 559,24 & $17,47 \%$ & $1.197,5$ & 527,93 & $18,81 \%$ & $5,93 \%$ \\
\hline & media - baja & $40,74 \%$ & $1.135,0$ & 399,68 & $31,12 \%$ & 963,4 & 345,12 & $17,81 \%$ & $15,81 \%$ \\
\hline & baja & $32,39 \%$ & $1.021,9$ & 389,36 & $35,26 \%$ & 819,2 & 283,85 & $24,75 \%$ & $37,17 \%$ \\
\hline \multirow{10}{*}{ sectores } & comercio & $20,72 \%$ & $1.242,1$ & 537,48 & $22,91 \%$ & 941,2 & 372,54 & $31,97 \%$ & $44,27 \%$ \\
\hline & mina e industria & $24,63 \%$ & $1.434,0$ & 619,47 & $14,29 \%$ & $1.095,2$ & 565,57 & $30,93 \%$ & $9,53 \%$ \\
\hline & construcción & $23,11 \%$ & $1.047,1$ & 354,07 & $2,07 \%$ & $1.055,4$ & 382,72 & $-0,79 \%$ & $-7,49 \%$ \\
\hline & hosteleria & $6,85 \%$ & $1.164,9$ & 502,59 & $9,93 \%$ & $1.047,6$ & 397,70 & $11,19 \%$ & $26,38 \%$ \\
\hline & $\begin{array}{l}\text { transporte y } \\
\text { telecom. }\end{array}$ & $7,60 \%$ & $1.529,6$ & 777,09 & $3,53 \%$ & $1.751,8$ & 762,02 & $-12,69 \%$ & $1,98 \%$ \\
\hline & $\begin{array}{l}\text { finanzas, } \\
\text { inmobiliaria, } \\
\text { investigación }\end{array}$ & $8,49 \%$ & $1.184,6$ & 543,40 & $20,67 \%$ & 941,8 & 389,76 & $25,78 \%$ & $39,42 \%$ \\
\hline & sector público & $2,37 \%$ & $1.322,6$ & 481,35 & $3,27 \%$ & $1.267,2$ & 573,27 & $4,37 \%$ & $-16,03 \%$ \\
\hline & educación & $2,27 \%$ & $1.550,8$ & 582,19 & $10,15 \%$ & $1.474,7$ & 551,61 & $5,16 \%$ & $5,54 \%$ \\
\hline & sanidad & $0,81 \%$ & $1.192,8$ & 438,21 & $6,89 \%$ & 944,3 & 373,68 & $26,32 \%$ & $17,27 \%$ \\
\hline & servicios & $3,15 \%$ & $1.020,2$ & 424,60 & $6,29 \%$ & 866,2 & 390,42 & $17,78 \%$ & $8,76 \%$ \\
\hline \multirow{6}{*}{ tamaño empresa } & $1 \cdot 10$ & $29,19 \%$ & $1.053,5$ & 459,49 & $30,83 \%$ & 894,9 & 399,11 & $17,73 \%$ & $15,13 \%$ \\
\hline & $11-50$ & $35,23 \%$ & $1.159,4$ & 480,87 & $33,38 \%$ & $1.086,4$ & 492,84 & $6,71 \%$ & $-2,43 \%$ \\
\hline & $51-100$ & $11,35 \%$ & $1.341,8$ & 573,29 & $10,29 \%$ & $1.149,9$ & 546,09 & $16,69 \%$ & $4,98 \%$ \\
\hline & $101-250$ & $16,24 \%$ & $1.558,2$ & 572,40 & $12,40 \%$ & $1.329,3$ & 514,75 & $17,22 \%$ & $11,20 \%$ \\
\hline & $251-500$ & $5,98 \%$ & $1.879,6$ & 667,84 & $6,03 \%$ & $1.394,0$ & 661,60 & $34,84 \%$ & $0,94 \%$ \\
\hline & $500-1000$ & $2,01 \%$ & $1.186,2$ & 544,22 & $7,08 \%$ & 840,0 & 278,45 & $41,21 \%$ & $95,45 \%$ \\
\hline \multirow{10}{*}{$\begin{array}{l}\text { antigüedad en el } \\
\text { puesto (contrato } \\
\text { actual) }\end{array}$} & $1-6$ meses & $33,47 \%$ & $1.029,1$ & 416,37 & $28,79 \%$ & 914,3 & 390,10 & $12,56 \%$ & $6,73 \%$ \\
\hline & $6-12$ meses & $12,80 \%$ & $1.218,8$ & 574,10 & $16,19 \%$ & $1.057,1$ & 541,14 & $15,30 \%$ & $6,09 \%$ \\
\hline & $12-18$ meses & $10,27 \%$ & $1.253,1$ & 505,88 & $8,63 \%$ & $1.053,8$ & 468,48 & $18,91 \%$ & $7,98 \%$ \\
\hline & 18 - 24 meses & $5,71 \%$ & $1.442,1$ & 694,67 & $7,74 \%$ & $1.186,8$ & 614,07 & $21,51 \%$ & $13,12 \%$ \\
\hline & $24-30$ meses & $4,98 \%$ & $1.277,9$ & 537,21 & $4,73 \%$ & $1.048,8$ & 472,37 & $21,84 \%$ & $13,73 \%$ \\
\hline & $30-36$ meses & $4,20 \%$ & $1.523,5$ & 704,61 & $5,72 \%$ & $1.233,7$ & 628,75 & $23,48 \%$ & $12,06 \%$ \\
\hline & $3-4$ años & $6,10 \%$ & $1.411,6$ & 598,69 & $7,36 \%$ & $1.117,5$ & 512,00 & $26,32 \%$ & $16,93 \%$ \\
\hline & 4 - 5 años & $4,09 \%$ & $1.456,8$ & 606,05 & $4,77 \%$ & $1.091,7$ & 485,93 & $33,44 \%$ & $24,72 \%$ \\
\hline & $5-6$ años & $3,22 \%$ & $1.526,3$ & 624,78 & $3,05 \%$ & $1.090,8$ & 476,51 & $39,93 \%$ & $31,12 \%$ \\
\hline & más 6 años & $15,15 \%$ & $1.474,8$ & 554,56 & $13,02 \%$ & $1.230,6$ & 488,79 & $19,84 \%$ & $13,46 \%$ \\
\hline \multirow{2}{*}{\multicolumn{2}{|c|}{ trabajando en una ETT }} & $9,56 \%$ & $1.591,1$ & 701,15 & $15,88 \%$ & $1.499,4$ & 639,92 & $6,12 \%$ & $9,57 \%$ \\
\hline & & $55,48 \%$ & $1.468,4$ & 614,53 & $60,59 \%$ & $1.205,2$ & 528,12 & $21,84 \%$ & $16,36 \%$ \\
\hline
\end{tabular}

Nota: La muestra de estimación contiene 32,322 salarios para hombres y 11,828 para mujeres. En total, cuenta con información sobre 1,657 empresas y 44,150 registros.

Fuente: Muestra del Fichero de Afiliados y Empresas del Régimen General de la Seguridad Social. Elaboración Propia. 
Cuadro 3. Distribución de los salarios mensuales por género (España).

\begin{tabular}{|c|c|c|c|c|c|c|c|c|c|c|}
\hline & & \multicolumn{3}{|c|}{ HOMBRES } & \multicolumn{3}{|c|}{ MUJERES } & \multirow{2}{*}{$\begin{array}{l}\text { diferencia } \\
\text { P75-25 }\end{array}$} & \multirow{2}{*}{$\begin{array}{l}\text { diferencia } \\
\text { P50-25 }\end{array}$} & \multirow{2}{*}{$\begin{array}{l}\text { diferencia } \\
\text { P75-50 }\end{array}$} \\
\hline & & P75-25 & P50-25 & P75-50 & P75-25 & P50-25 & P75-50 & & & \\
\hline \multirow{2}{*}{\multicolumn{2}{|c|}{ muestra completa }} & 747,0 & 249,4 & 497,7 & 599,2 & 238,5 & 360,7 & $24,68 \%$ & $4,58 \%$ & $37,96 \%$ \\
\hline & $16-29$ & 442,1 & 175,7 & 266,4 & 444,4 & 190,6 & 253,9 & $-0,53 \%$ & $.7,81 \%$ & $4,93 \%$ \\
\hline \multirow[t]{2}{*}{ edad } & $30-45$ & 800,8 & 263,5 & 537,4 & 731,2 & 281,6 & 449,6 & $9,52 \%$ & $-6,44 \%$ & $19,52 \%$ \\
\hline & $46-65$ & 986,7 & 313,9 & 672,7 & 667,2 & 271,2 & 396,0 & $47,88 \%$ & $15,73 \%$ & $69,90 \%$ \\
\hline \multicolumn{2}{|c|}{ inmigrante } & 363,4 & 166,7 & 196,7 & 378,5 & 180,8 & 197,8 & $-4,00 \%$ & $-7,79 \%$ & $-0,53 \%$ \\
\hline \multirow{5}{*}{ cualificación } & alta & $1.146,7$ & 773,2 & 373,4 & 821,2 & 464,2 & 356,9 & $39,64 \%$ & $66,56 \%$ & $4,62 \%$ \\
\hline & media - alta & $1.279,0$ & 600,6 & 678,4 & $1.179,4$ & 377,5 & 801,9 & $8,44 \%$ & $59,09 \%$ & $-15,40 \%$ \\
\hline & media & 832,4 & 276,9 & 555,4 & 636,0 & 270,1 & 365,9 & $30,87 \%$ & $2,51 \%$ & $51,81 \%$ \\
\hline & media - baja & 543,1 & 201,9 & 341,2 & 448,4 & 206,7 & 241,7 & $21,13 \%$ & $-2,34 \%$ & $41,21 \%$ \\
\hline & baja & 407,3 & 160,3 & 246,9 & 419,7 & 220,2 & 199,5 & $-2,95 \%$ & $-27,18 \%$ & $23,78 \%$ \\
\hline \multirow{10}{*}{ sectores } & comercio & 658,5 & 241,5 & 417,0 & 430,2 & 142,5 & 287,7 & $53,06 \%$ & $69,47 \%$ & $44,93 \%$ \\
\hline & mina e industria & 853,7 & 298,2 & 555,5 & 503,1 & 201,4 & 301,8 & $69,67 \%$ & $48,06 \%$ & $84,09 \%$ \\
\hline & construcción & 417,0 & 140,1 & 277,0 & 495,1 & 171,0 & 324,1 & $-15,77 \%$ & $-18,06 \%$ & $-14,55 \%$ \\
\hline & $\begin{array}{l}\text { hosteleria } \\
\text { hen }\end{array}$ & 442,9 & 187,9 & 255,0 & 372,1 & 204,5 & 167,6 & $19,02 \%$ & $-8,12 \%$ & $52,14 \%$ \\
\hline & $\begin{array}{l}\text { transporte y telecom. } \\
\text { finanzas, }\end{array}$ & $1.023,1$ & 296,9 & 726,2 & 791,0 & 294,3 & 496,7 & $29,35 \%$ & $0,88 \%$ & $46,22 \%$ \\
\hline & $\begin{array}{l}\text { inmobiliaria, } \\
\text { investigación }\end{array}$ & $1.389,4$ & 388,3 & $1.001,1$ & 685,7 & 258,4 & 427,3 & $102,62 \%$ & $50,25 \%$ & $134,29 \%$ \\
\hline & sector público & 354,7 & 162,5 & 192,3 & 793,6 & 253,7 & 539,9 & $-55,30 \%$ & $-35,95 \%$ & $-64,39 \%$ \\
\hline & educación & 923,3 & 411,0 & 512,4 & 925,5 & 517,8 & 407,7 & $-0,24 \%$ & $-20,64 \%$ & $25,68 \%$ \\
\hline & sanidad & 776,4 & 317,0 & 459,4 & 617,8 & 278,5 & 339,3 & $25,68 \%$ & $13,84 \%$ & $35,39 \%$ \\
\hline & servicios & 613,5 & 293,2 & 320,3 & 568,1 & 252,5 & 315,7 & $7,98 \%$ & $16,12 \%$ & $1,47 \%$ \\
\hline \multirow{6}{*}{ tamaño empresa } & $1 \cdot 10$ & 415,6 & 164,5 & 251,1 & 483,4 & 217,4 & 266,0 & $-14,03 \%$ & $-24,36 \%$ & $-5,59 \%$ \\
\hline & $11-50$ & 556,2 & 204,0 & 352,2 & 621,0 & 232,0 & 389,0 & $-10,43 \%$ & $.12,05 \%$ & $-9,47 \%$ \\
\hline & $51-100$ & 877,3 & 327,3 & 550,0 & 692,0 & 283,6 & 408,4 & $26,78 \%$ & $15,43 \%$ & $34,67 \%$ \\
\hline & $101-250$ & 901,4 & 321,4 & 580,1 & 627,4 & 247,4 & 380,0 & $43,67 \%$ & $29,87 \%$ & $52,66 \%$ \\
\hline & $251 \cdot 500$ & $1.337,0$ & 528,3 & 808,7 & 724,9 & 265,8 & 459,1 & $84,44 \%$ & $98,78 \%$ & $76,13 \%$ \\
\hline & $500 \cdot 1000$ & $1.016,9$ & 623,4 & 393,5 & 695,1 & 288,4 & 406,7 & $46,30 \%$ & $116,17 \%$ & $-3,24 \%$ \\
\hline \multirow{10}{*}{$\begin{array}{l}\text { antigüedad en el } \\
\text { puesto (contrato } \\
\text { actual) }\end{array}$} & 1.6 meses & 472,8 & 195,5 & 277,3 & 477,1 & 204,6 & 272,6 & $-0,91 \%$ & $-4,43 \%$ & $1,72 \%$ \\
\hline & 6 - 12 meses & 511,3 & 177,4 & 333,9 & 519,2 & 184,2 & 335,0 & $-1,53 \%$ & $-3,72 \%$ & $-0,33 \%$ \\
\hline & $12 \cdot 18$ meses & 727,9 & 239,0 & 489,0 & 552,3 & 218,5 & 333,8 & $31,81 \%$ & $9,37 \%$ & $46,50 \%$ \\
\hline & $18 \cdot 24$ meses & 725,7 & 223,4 & 502,3 & 637,1 & 231,3 & 405,8 & $13,91 \%$ & $-3,40 \%$ & $23,78 \%$ \\
\hline & $24-30$ meses & 746,3 & 247,0 & 499,2 & 548,8 & 221,8 & 327,0 & $35,99 \%$ & $11,36 \%$ & $52,69 \%$ \\
\hline & $30-36$ meses & 805,9 & 253,4 & 552,6 & 654,3 & 242,7 & 411,6 & $23,17 \%$ & $4,40 \%$ & $34,23 \%$ \\
\hline & 3.4 años & 854,4 & 281,4 & 573,0 & 620,5 & 236,5 & 383,9 & $37,71 \%$ & $18,99 \%$ & $49,24 \%$ \\
\hline & $4-5$ años & 893,0 & 292,9 & 600,1 & 689,0 & 266,2 & 422,9 & $29,60 \%$ & $10,06 \%$ & $41,90 \%$ \\
\hline & $5-6$ años & 955,2 & 296,4 & 658,8 & 763,3 & 265,0 & 498,3 & $25,15 \%$ & $11,86 \%$ & $32,22 \%$ \\
\hline & más 6 años & 920,6 & 303,0 & 617,6 & 785,1 & 270,2 & 515,0 & $17,26 \%$ & $12,15 \%$ & $19,94 \%$ \\
\hline \multirow{17}{*}{ ccaa } & Andalucía & 549,6 & 191,0 & 358,6 & 571,4 & 234,0 & 337,3 & $-3,81 \%$ & $-18,38 \%$ & $6,30 \%$ \\
\hline & Aragón & 730,9 & 325,7 & 405,2 & 553,6 & 182,0 & 371,6 & $32,02 \%$ & $78,93 \%$ & $9,04 \%$ \\
\hline & Asturias & $1.074,8$ & 611,8 & 463,0 & 969,5 & 308,2 & 661,4 & $10,86 \%$ & $98,55 \%$ & $-30,00 \%$ \\
\hline & Baleares & $1.085,7$ & 226,1 & 859,5 & 439,7 & 190,0 & 249,7 & $146,92 \%$ & $19,02 \%$ & $244,23 \%$ \\
\hline & Canarias & 550,4 & 186,7 & 363,7 & 539,0 & 184,8 & 354,2 & $2,12 \%$ & $1,06 \%$ & $2,67 \%$ \\
\hline & Cantabria & 513,8 & 171,2 & 342,7 & 732,1 & 330,5 & 401,6 & $-29,81 \%$ & $-48,20 \%$ & $-14,67 \%$ \\
\hline & Castilla-León & 342,1 & 141,8 & 200,3 & 432,4 & 168,4 & 264,0 & $-20,89 \%$ & $-15,80 \%$ & $-24,13 \%$ \\
\hline & Castilla-LM & 411,9 & 170,7 & 241,2 & 534,9 & 233,4 & 301,5 & $-23,00 \%$ & $-26,87 \%$ & $-20,00 \%$ \\
\hline & Cataluña & 900,1 & 272,2 & 627,9 & 667,7 & 241,7 & 426,0 & $34,80 \%$ & $12,59 \%$ & $47,41 \%$ \\
\hline & Valencia & 415,8 & 126,7 & 289,2 & 409,4 & 172,4 & 236,9 & $1,58 \%$ & $-26,55 \%$ & $22,06 \%$ \\
\hline & Extremadura & 360,5 & 105,5 & 255,0 & 606,4 & 283,6 & 322,7 & $.40,54 \%$ & $-62,80 \%$ & $-20,98 \%$ \\
\hline & Galicia & 504,1 & 144,1 & 360,0 & 520,7 & 130,3 & 390,4 & $-3,18 \%$ & $10,60 \%$ & $-7,78 \%$ \\
\hline & Madrid & 940,9 & 313,4 & 627,6 & 820,0 & 300,5 & 519,5 & $14,75 \%$ & $4,28 \%$ & $20,80 \%$ \\
\hline & Murcia & 461,4 & 124,4 & 336,9 & 516,4 & 222,0 & 294,5 & $-10,66 \%$ & $-43,94 \%$ & $14,42 \%$ \\
\hline & Navarra & 879,3 & 315,0 & 564,3 & 665,5 & 272,5 & 392,9 & $32,13 \%$ & $15,57 \%$ & $43,62 \%$ \\
\hline & $\begin{array}{l}\text { P. Vasco } \\
\text { P }\end{array}$ & 803,1 & 266,1 & 537,1 & 669,0 & 292,3 & 376,7 & $20,06 \%$ & $-8,96 \%$ & $42,57 \%$ \\
\hline & Rioja & 634,9 & 311,1 & 323,8 & 682,2 & 277,9 & 404,4 & $-6,93 \%$ & $11,97 \%$ & $-19,92 \%$ \\
\hline \multirow{2}{*}{\multicolumn{2}{|c|}{$\begin{array}{l}\text { trabajando en una ETT } \\
\text { contrato indefinido }\end{array}$}} & 826,5 & 250,0 & 576,5 & 845,8 & 346,5 & 499,3 & $-2,29 \%$ & $-27,87 \%$ & $15,46 \%$ \\
\hline & & 906,7 & 293,5 & 613,2 & 675,4 & 242,5 & 432,9 & $34,26 \%$ & $21,06 \%$ & $41,65 \%$ \\
\hline
\end{tabular}

Fuente: Muestra del Fichero de Afiliados y Empresas del Régimen General de la Seguridad Social. Elaboración Propia. 
Cuadro 4. Distribución de los salarios mensuales por género (Andalucía)

\begin{tabular}{|c|c|c|c|c|c|c|c|c|c|c|}
\hline & \multicolumn{3}{|c|}{ HOMBRES } & \multicolumn{3}{|c|}{ MUJERES } & \multirow{2}{*}{$\begin{array}{l}\text { diferencias } \\
\text { P75-25 }\end{array}$} & \multirow{2}{*}{$\begin{array}{c}\text { diferencias } \\
\text { P75-50 }\end{array}$} & \multirow{2}{*}{$\begin{array}{l}\text { diferencias } \\
\text { P50-25 }\end{array}$} \\
\hline & & P75-25 & P75-50 & P50-25 & P75-25 & P75-50 & P50-25 & & & \\
\hline \multicolumn{2}{|c|}{ muestra completa } & 549,6 & 358,6 & 191,0 & 571,4 & 337,3 & 234,0 & $-3,81 \%$ & $6,30 \%$ & $-18,38 \%$ \\
\hline \multirow{3}{*}{ edad } & $16 \cdot 29$ & 378,2 & 210,1 & 168,0 & 469,3 & 240,2 & 229,1 & $-19,42 \%$ & $-12,52 \%$ & $-26,66 \%$ \\
\hline & $30-45$ & 620,3 & 414,8 & 205,5 & 609,3 & 359,9 & 249,4 & $1,81 \%$ & $15,24 \%$ & $-17,58 \%$ \\
\hline & $46 \cdot 65$ & 806,2 & 534,9 & 271,3 & 641,7 & 460,4 & 181,3 & $25,64 \%$ & $16,18 \%$ & $49,66 \%$ \\
\hline \multicolumn{2}{|c|}{ inmigrante } & 253,4 & 125,5 & 127,9 & 413,5 & 146,2 & 267,4 & $-38,73 \%$ & $-14,17 \%$ & $-52,15 \%$ \\
\hline \multirow{5}{*}{ cualificación } & alta & $1.230,7$ & 594,2 & 636,5 & 690,3 & 284,9 & 405,4 & $78,29 \%$ & $108,59 \%$ & $57,01 \%$ \\
\hline & media - alta & $1.285,0$ & 556,1 & 728,9 & $1.321,1$ & 974,2 & 346,9 & $-2,73 \%$ & $-42,92 \%$ & $110,15 \%$ \\
\hline & media & 761,7 & 491,5 & 270,2 & 580,2 & 347,5 & 232,7 & $31,28 \%$ & $41,45 \%$ & $16,11 \%$ \\
\hline & media - baja & 376,6 & 235,6 & 141,0 & 428,2 & 226,8 & 201,4 & $-12,07 \%$ & $3,86 \%$ & $-30,00 \%$ \\
\hline & baja & 383,7 & 221,0 & 162,7 & 392,4 & 152,9 & 239,5 & $-2,20 \%$ & $44,52 \%$ & $-32,04 \%$ \\
\hline \multirow{11}{*}{ sectores } & comercio & 517,7 & 317,0 & 200,7 & 395,4 & 208,3 & 187,1 & $30,91 \%$ & $52,17 \%$ & $7,24 \%$ \\
\hline & mina e industria & 847,9 & 545,7 & 302,2 & 738,8 & 378,3 & 360,5 & $14,76 \%$ & $44,23 \%$ & $-16,17 \%$ \\
\hline & construcción & 244,8 & 155,4 & 89,4 & 351,6 & 205,5 & 146,1 & $-30,37 \%$ & $-24,36 \%$ & $-38,82 \%$ \\
\hline & hosteleria & 477,6 & 316,0 & 161,6 & 403,1 & 218,4 & 184,7 & $18,50 \%$ & $44,72 \%$ & $-12,51 \%$ \\
\hline & $\begin{array}{l}\text { transporte y } \\
\text { telecom. }\end{array}$ & $1.635,9$ & $1.284,9$ & 351,0 & $1.524,7$ & 459,0 & $1.065,8$ & $7,29 \%$ & $179,96 \%$ & $-67,07 \%$ \\
\hline & finanzas, & & & & & & & & & \\
\hline & $\begin{array}{l}\text { inmobiliaria, } \\
\text { investigación }\end{array}$ & 525,3 & 325,0 & 200,3 & 409,0 & 230,3 & 178,7 & $28,44 \%$ & $41,11 \%$ & $12,10 \%$ \\
\hline & sector público & 376,8 & 158,7 & 218,1 & 674,2 & 332,0 & 342,2 & $-44,11 \%$ & $-52,19 \%$ & $-36,28 \%$ \\
\hline & educación & 990,5 & 514,2 & 476,3 & 966,7 & 317,7 & 649,0 & $2,46 \%$ & $61,87 \%$ & $-26,61 \%$ \\
\hline & sanidad & 539,5 & 167,8 & 371,6 & 608,3 & 466,0 & 142,3 & $-11,32 \%$ & $-63,98 \%$ & $161,08 \%$ \\
\hline & servicios & 547,3 & 270,9 & 276,4 & 483,2 & 309,6 & 173,6 & $13,27 \%$ & $-12,51 \%$ & $59,25 \%$ \\
\hline \multirow{6}{*}{ tamaño empresa } & $1-10$ & 360,1 & 196,7 & 163,4 & 488,9 & 231,7 & 257,2 & $-26,35 \%$ & $-15,14 \%$ & $-36,46 \%$ \\
\hline & $11 \cdot 50$ & 386,8 & 236,4 & 150,3 & 576,3 & 368,9 & 207,4 & $-32,88 \%$ & $-35,91 \%$ & $-27,51 \%$ \\
\hline & $51 \cdot 100$ & 693,6 & 444,4 & 249,2 & 556,7 & 400,0 & 156,7 & $24,58 \%$ & $11,10 \%$ & $59,00 \%$ \\
\hline & $101-250$ & 737,4 & 490,5 & 246,9 & 420,0 & 237,0 & 183,0 & $75,57 \%$ & $106,99 \%$ & $34,89 \%$ \\
\hline & $251-500$ & $1.258,6$ & 712,0 & 546,5 & $1.033,6$ & 734,9 & 298,7 & $21,76 \%$ & $-3,12 \%$ & $83,00 \%$ \\
\hline & $500-1000$ & 515,0 & 395,9 & 119,1 & 300,2 & 78,6 & 221,5 & $71,59 \%$ & $403,60 \%$ & $-46,23 \%$ \\
\hline \multirow{11}{*}{$\begin{array}{l}\text { antigüedad en el } \\
\text { puesto (contrato } \\
\text { actual) }\end{array}$} & $1-6$ meses & 413,2 & 221,6 & 191,6 & 568,6 & 295,0 & 273,6 & $-27,33 \%$ & $-24,89 \%$ & $-29,96 \%$ \\
\hline & $6 \cdot 12$ meses & 481,1 & 333,1 & 148,0 & 599,0 & 380,3 & 218,7 & $-19,68 \%$ & $-12,40 \%$ & $-32,33 \%$ \\
\hline & $12 \cdot 18$ meses & 509,1 & 317,4 & 191,7 & 515,3 & 313,1 & 202,2 & $-1,21 \%$ & $1,37 \%$ & $-5,20 \%$ \\
\hline & $18 \cdot 24$ meses & $1.053,3$ & 809,0 & 244,3 & 800,0 & 581,6 & 218,4 & $31,66 \%$ & $39,09 \%$ & $11,88 \%$ \\
\hline & $24 \cdot 30$ meses & 560,5 & 376,8 & 183,7 & 472,4 & 297,4 & 175,0 & $18,66 \%$ & $26,73 \%$ & $4,96 \%$ \\
\hline & $30-36$ meses & $1.294,3$ & $1.012,3$ & 282,1 & 880,5 & 697,1 & 183,3 & $47,00 \%$ & $45,20 \%$ & $53,86 \%$ \\
\hline & $3 \cdot 4$ años & 789,4 & 520,2 & 269,2 & 564,0 & 394,7 & 169,3 & $39,95 \%$ & $31,77 \%$ & $59,01 \%$ \\
\hline & 4 - 5 años & 938,5 & 635,6 & 302,9 & 547,6 & 408,8 & 138,7 & $71,39 \%$ & $55,47 \%$ & $118,28 \%$ \\
\hline & $5 \cdot 6$ años & $1.033,5$ & 707,5 & 326,0 & 520,7 & 331,7 & 189,0 & $98,48 \%$ & $113,30 \%$ & $72,48 \%$ \\
\hline & más 6 años & 701,1 & 452,9 & 248,2 & 508,4 & 295,9 & 212,5 & $37,92 \%$ & $53,06 \%$ & $16,83 \%$ \\
\hline & & $1.247,7$ & 971,4 & 276,2 & 951,1 & 483,5 & 467,7 & $31,17 \%$ & $100,92 \%$ & $-40,93 \%$ \\
\hline \multicolumn{2}{|c|}{ contrato indefinido } & 873,9 & 590,3 & 283,6 & 598,4 & 381,6 & 216,8 & $46,03 \%$ & $54,69 \%$ & $30,79 \%$ \\
\hline
\end{tabular}

Fuente: Muestra del Fichero de Afiliados y Empresas del Régimen General de la Seguridad Social. Elaboración Propia. 
Cuadro 5. Regresión censurada con estimación de la varianza: España. Variable dependiente: logaritmo del salario mensual.

\begin{tabular}{|c|c|c|c|c|c|c|c|c|}
\hline & \multicolumn{4}{|c|}{ HOMBRES } & \multicolumn{4}{|c|}{ MUJERES } \\
\hline & \multicolumn{2}{|c|}{ Media } & \multicolumn{2}{|c|}{ D. típica } & \multicolumn{2}{|c|}{ Media } & \multicolumn{2}{|c|}{ D. típica } \\
\hline & Coeficiente & $\mathrm{t}$ & Coeficiente & $\mathrm{t}$ & Coeficiente & $\mathrm{t}$ & Coeficiente & $\mathrm{t}$ \\
\hline edad & 0,0225 & 49,56 & $-0,0084$ & $-7,59$ & 0,0188 & 28,12 & 0,0093 & 5,73 \\
\hline edad $^{2}$ & $-0,0002$ & $-38,60$ & 0,0001 & 8,70 & $-0,0002$ & $-24,76$ & $-0,0001$ & $-3,29$ \\
\hline inmigrante & $-0,0318$ & $-4,79$ & $-0,0266$ & $-1,72$ & 0,0440 & 3,09 & 0,0049 & 0,17 \\
\hline cualificación alta & 0,7047 & 153,73 & 0,5287 & 59,09 & 0,6443 & 140,48 & 0,3084 & 30,19 \\
\hline cualificación media - alta & 0,3253 & 34,08 & 0,2767 & 15,44 & 0,4208 & 30,63 & 0,2719 & 10,64 \\
\hline cualificación media & 0,0711 & 10,22 & 0,1327 & 9,62 & 0,2583 & 37,83 & 0,0932 & 6,48 \\
\hline cualificación media - baja & 0,0895 & 54,23 & $-0,0004$ & $-0,09$ & 0,1438 & 54,27 & 0,0244 & 3,70 \\
\hline mina e industria & 0,0423 & 19,23 & $-0,1000$ & $-18,96$ & 0,0740 & 22,99 & 0,1057 & 13,59 \\
\hline construcción & 0,0218 & 9,13 & $-0,1879$ & $-32,23$ & 0,1078 & 16,91 & $-0,0337$ & $-2,14$ \\
\hline hosteleria & $-0,1107$ & $-30,15$ & $-0,1164$ & $-12,99$ & 0,0573 & 13,42 & 0,0034 & 0,32 \\
\hline transporte y telecom. & 0,0505 & 14,97 & $-0,0148$ & $-1,86$ & 0,2039 & 30,78 & 0,1182 & 7,75 \\
\hline finanzas, inmobiliaria, investigación & $-0,0253$ & $-7,21$ & 0,1526 & 21,22 & 0,0131 & 3,80 & 0,1929 & 24,09 \\
\hline sector público & 0,0294 & 2,36 & $-0,1564$ & $-6,09$ & 0,0972 & 6,90 & $-0,2600$ & $-9,44$ \\
\hline educación & $-0,1621$ & $-11,84$ & $-0,0768$ & $-3,02$ & 0,0791 & 7,33 & 0,0370 & 1,67 \\
\hline sanidad & $-0,2186$ & $-28,13$ & $-0,0905$ & $-5,11$ & $-0,1140$ & $-27,62$ & $-0,0656$ & $-6,39$ \\
\hline servicios & $-0,1336$ & $-27,13$ & 0,1308 & 12,71 & $-0,1009$ & $-21,54$ & 0,1080 & 9,60 \\
\hline tamaño $11-50$ & 0,1118 & 62,61 & $-0,0157$ & $-3,57$ & 0,1224 & 42,88 & $-0,0786$ & $-12,06$ \\
\hline tamaño $51-100$ & 0,2246 & 88,98 & 0,0747 & 12,65 & 0,2010 & 56,15 & $-0,0960$ & $-11,61$ \\
\hline tamaño $101-250$ & 0,2307 & 61,41 & 0,0090 & 1,06 & 0,2707 & 45,93 & $-0,1251$ & $-9,88$ \\
\hline tamaño $251-500$ & 0,2311 & 29,59 & 0,0165 & 1,01 & 0,2642 & 39,13 & $-0,0953$ & $-6,32$ \\
\hline tamaño $501-1000$ & 0,3446 & 46,32 & $-0,0711$ & $-4,40$ & 0,2673 & 40,19 & $-0,2190$ & $-15,05$ \\
\hline antigüedad entre $6 \cdot 12$ meses & 0,0803 & 31,08 & $-0,1887$ & $-31,58$ & 0,0382 & 11,00 & $-0,1040$ & $-12,98$ \\
\hline antigüedad entre $12-18$ meses & 0,1069 & 39,21 & $-0,1977$ & $-30,79$ & 0,0797 & 20,87 & $-0,1235$ & $-13,60$ \\
\hline antigüedad entre 18 - 24 meses & 0,0893 & 27,37 & $-0,1967$ & $-25,10$ & 0,0687 & 15,13 & $-0,0885$ & $-8,37$ \\
\hline antigüedad entre 24 - 30 meses & 0,0972 & 29,43 & $-0,2202$ & $-27,38$ & 0,0836 & 18,96 & $-0,1760$ & $-16,10$ \\
\hline antigüedad entre $30-36$ meses & 0,0838 & 22,85 & $-0,2306$ & $-25,57$ & 0,0700 & 13,80 & $-0,1224$ & $-10,02$ \\
\hline antigüedad entre $3-4$ años & 0,1060 & 32,51 & $-0,1840$ & $-23,78$ & 0,0877 & 21,03 & $-0,1447$ & $-14,14$ \\
\hline antigüedad entre 4 - 5 años & 0,1204 & 34,45 & $-0,2018$ & $-23,51$ & 0,1121 & 23,73 & $-0,1342$ & $-11,60$ \\
\hline antigüedad entre $5 \cdot 6$ años & 0,1351 & 36,24 & $-0,2429$ & $-25,94$ & 0,1405 & 26,82 & $-0,1412$ & $-10,82$ \\
\hline antigüedad entre más 6 años & 0,1763 & 68,30 & $-0,2579$ & $-42,89$ & 0,1877 & 53,30 & $-0,1333$ & $-16,35$ \\
\hline trabajando en una ETT & $-0,0561$ & $-7,24$ & 0,1143 & 6,41 & 0,0715 & 5,97 & 0,2270 & 10,03 \\
\hline cualificación medio-alto * ETT & $-0,1002$ & $-10,44$ & $-0,2102$ & $-9,03$ & $-0,1594$ & $-13,64$ & $-0,2541$ & $-8,75$ \\
\hline cualificación medio * ETT & 0,0280 & 3,30 & $-0,0631$ & $-2,95$ & $-0,0086$ & $-1,18$ & $-0,1815$ & $-9,26$ \\
\hline cualificación medio-bajo * ETT & 0,0214 & 3,31 & $-0,0125$ & $-0,69$ & 0,0695 & 9,56 & $-0,0726$ & $-4,06$ \\
\hline hosteleria * ETT & 0,2786 & 14,98 & $-0,0964$ & $-1,95$ & $-0,0423$ & $-1,47$ & 0,0519 & 0,83 \\
\hline tpte y telecom * ETT & 0,1762 & 13,13 & $-0,1576$ & $-4,79$ & 0,3243 & 16,13 & $-0,6884$ & $-9,02$ \\
\hline educacion * ETT & 0,1154 & 8,38 & $-0,1249$ & $-4,56$ & 0,0643 & 4,58 & $-0,1908$ & $-6,82$ \\
\hline sanidad * ETT & 0,0985 & 7,48 & $-0,2669$ & $-8,66$ & 0,1040 & 8,08 & $-0,2148$ & $-8,29$ \\
\hline servicios * ETT & 0,4474 & 21,56 & $-0,0297$ & $-0,78$ & 0,4505 & 21,94 & $-0,0568$ & $-1,45$ \\
\hline contrato indefinido & 0,1015 & 44,87 & $-0,1317$ & $-24,34$ & 0,2290 & 61,29 & $-0,2889$ & $-35,33$ \\
\hline inmigrante * C.I. & $-0,0767$ & $-8,05$ & $-0,0164$ & $-0,71$ & $-0,1001$ & $-5,40$ & $-0,0077$ & $-0,18$ \\
\hline cualificacion medio - alto * C.I. & 0,1537 & 15,47 & 0,1047 & 5,54 & 0,1415 & 10,09 & 0,1779 & 6,60 \\
\hline cualificacion medio ${ }^{*}$ C.I. & 0,1371 & 19,07 & 0,0759 & 5,25 & 0,0205 & 2,93 & 0,0554 & 3,67 \\
\hline sector público * C.I. & $-0,1157$ & $-10,23$ & $-0,1504$ & $-5,76$ & $-0,1387$ & $-13,87$ & 0,0208 & 0,84 \\
\hline educacion * C.I. & $-0,1403$ & $-10,61$ & 0,1173 & 4,33 & $-0,2025$ & $-19,42$ & 0,0192 & 0,87 \\
\hline tamaño $101-250 *$ C.I. & 0,0474 & 11,22 & $-0,0086$ & $-0,89$ & $-0,0013$ & $-0,21$ & 0,0260 & 1,81 \\
\hline tamaño $251-500 *$ C.I. & 0,1185 & 13,93 & $-0,0742$ & $-4,00$ & 0,0035 & 0,49 & $-0,1956$ & $-11,46$ \\
\hline tamaño $501-1000 *$ C.I. & 0,0339 & 4,44 & $-0,2836$ & $-15,74$ & $-0,0024$ & $-0,33$ & $-0,2505$ & $-14,11$ \\
\hline Constante & 6,1667 & 678,89 & $-0,7277$ & $-33,70$ & 5,9974 & 460,35 & $-1,0764$ & $-34,41$ \\
\hline Observaciones & 228.940 & & & & 123.098 & & & \\
\hline Log likelihood & $-91.673,01$ & & & & $-54.791,58$ & & & \\
\hline
\end{tabular}

Grupo de referencia: trabajador de una empresa dedicada al comercio de menos 10 empleados, no inmigrante, con baja cualificación, de menos de 6 meses de antigüedad en el puesto de trabajo, no contratado por una ETT, sin trabajo fijo y residente en la comunidad de Madrid. 
Cuadro 6. Regresión censurada con estimación de la varianza: Andalucía. Variable dependiente: logaritmo del salario mensual.

\begin{tabular}{|c|c|c|c|c|c|c|c|c|}
\hline & \multicolumn{4}{|c|}{ HOMBRES } & \multicolumn{4}{|c|}{ MUJERES } \\
\hline & \multicolumn{2}{|c|}{ Media } & \multicolumn{2}{|c|}{ D. típica } & \multicolumn{2}{|c|}{ Media } & \multicolumn{2}{|c|}{ D. típica } \\
\hline & Coeficiente & $\bar{t}$ & Coeficiente & $\bar{t}$ & Coeficiente & $\bar{t}$ & Coeficiente & $\bar{t}$ \\
\hline edad & 0,0254 & 20,77 & $-0,0227$ & $-7,55$ & 0,0188 & 9,10 & 0,0165 & 2,94 \\
\hline edad $^{2}$ & $-0,0002$ & $-16,29$ & 0,0003 & 7,42 & $-0,0002$ & $-7,76$ & $-0,0002$ & $-2,79$ \\
\hline inmigrante & $-0,1003$ & $-3,85$ & $-0,1664$ & $-2,18$ & 0,0707 & 0,89 & 0,0645 & 0,39 \\
\hline cualificación alta & 0,5910 & 46,05 & 0,5294 & 20,72 & 0,6093 & 38,32 & 0,3658 & 10,50 \\
\hline cualificación media - alta & 0,2962 & 12,19 & 0,3094 & 6,09 & 0,3944 & 7,09 & 0,2046 & 1,86 \\
\hline cualificación media & 0,0767 & 4,28 & 0,2582 & 7,48 & 0,2182 & 9,00 & 0,2874 & 6,38 \\
\hline cualificación media - baja & 0,0550 & 12,71 & $-0,0562$ & $-4,97$ & 0,1565 & 17,84 & 0,0240 & 1,07 \\
\hline mina e industria & 0,0346 & 5,90 & 0,0699 & 4,77 & 0,0637 & 4,78 & 0,3532 & 12,29 \\
\hline construcción & 0,0164 & 2,73 & $-0,1027$ & $-6,68$ & 0,0412 & 1,79 & 0,0119 & 0,22 \\
\hline hosteleria & $-0,0904$ & $-11,53$ & $-0,0576$ & $-2,81$ & 0,0954 & 7,92 & $-0,0561$ & $-1,81$ \\
\hline transporte y telecom. & $-0,0091$ & $-1,02$ & 0,0565 & 2,62 & 0,1639 & 6,98 & 0,0250 & 0,47 \\
\hline finanzas, inmobiliaria, investigación & $-0,1178$ & $-11,37$ & 0,3319 & 15,47 & $-0,0387$ & $-3,30$ & 0,1050 & 3,70 \\
\hline sector público & 0,0521 & 1,69 & 0,6016 & 10,22 & 0,0674 & 1,07 & 0,3661 & 3,63 \\
\hline educación & $-0,2297$ & $-4,60$ & 0,1603 & 1,85 & 0,0542 & 1,45 & 0,1451 & 2,05 \\
\hline sanidad & $-0,0978$ & $-5,44$ & $-0,1340$ & $-2,64$ & $-0,0887$ & $-6,77$ & $-0,0768$ & $-2,08$ \\
\hline servicios & $-0,1926$ & $-15,30$ & 0,0865 & 2,93 & $-0,1187$ & $-7,80$ & 0,0208 & 0,56 \\
\hline tamaño $11 \cdot 50$ & 0,1037 & 22,65 & $-0,0991$ & $-8,71$ & 0,1191 & 13,50 & $-0,1236$ & $-5,92$ \\
\hline tamaño $51-100$ & 0,2441 & 34,24 & 0,0530 & 3,22 & 0,2596 & 21,67 & $-0,2104$ & $-6,90$ \\
\hline tamaño $101-250$ & 0,3064 & 36,21 & $-0,1007$ & $-4,81$ & 0,4725 & 24,08 & $-0,2347$ & $-5,43$ \\
\hline tamaño $251-500$ & 0,5801 & 26,00 & 0,0889 & 1,99 & 0,4113 & 15,17 & $-0,2756$ & $-4,40$ \\
\hline tamaño $501-1000$ & $-0,0001$ & 0,00 & $-0,4079$ & $-5,20$ & 0,0051 & 0,17 & $-0,0596$ & $-0,87$ \\
\hline antigüedad entre 6 - 12 meses & 0,0616 & 10,22 & $-0,1822$ & $-12,03$ & 0,0517 & 4,85 & $-0,0738$ & $-2,85$ \\
\hline antigüedad entre $12-18$ meses & 0,0716 & 11,35 & $-0,2180$ & $-13,28$ & 0,0490 & 3,98 & $-0,0468$ & $-1,51$ \\
\hline antigüedad entre 18 - 24 meses & 0,0879 & 10,26 & $-0,1655$ & $-7,65$ & 0,0626 & 5,10 & $-0,1346$ & $-4,04$ \\
\hline antigüedad entre 24 - 30 meses & 0,0723 & 8,58 & $-0,2090$ & $-9,47$ & 0,0536 & 3,72 & $-0,1283$ & $-3,30$ \\
\hline antigüedad entre $30-36$ meses & 0,0736 & 7,73 & $-0,1733$ & $-6,89$ & 0,0371 & 2,82 & $-0,1719$ & $-4,57$ \\
\hline antigüedad entre 3 - 4 años & 0,1101 & 12,90 & $-0,1542$ & $-7,10$ & 0,0856 & 6,69 & $-0,1034$ & $-3,07$ \\
\hline antigüedad entre $4-5$ años & 0,1276 & 13,07 & $-0,1870$ & $-7,52$ & 0,0899 & 6,23 & $-0,1563$ & $-4,05$ \\
\hline antigüedad entre 5 - 6 años & 0,1477 & 14,31 & $-0,2369$ & $-8,60$ & 0,0916 & 5,19 & $-0,0949$ & $-2,04$ \\
\hline antigüedad entre más 6 años & 0,1469 & 21,41 & $-0,2438$ & $-14,14$ & 0,1810 & 14,67 & $-0,1009$ & $-3,29$ \\
\hline trabajando en una ETT & $-0,1096$ & $-8,12$ & $-0,2956$ & $-7,95$ & $-0,0869$ & $-3,56$ & 0,2118 & 3,81 \\
\hline cualificación media - alta * ETT & $-0,0460$ & $-2,03$ & $-0,6102$ & $-8,90$ & $-0,1226$ & $-3,39$ & $-0,0919$ & $-0,80$ \\
\hline cualificación media * ETT & 0,0286 & 1,62 & $-0,0150$ & $-0,29$ & $-0,0049$ & $-0,18$ & $-0,2893$ & $-3,88$ \\
\hline cualificación media - baja* ETT & 0,0830 & 5,82 & 0,1669 & 4,11 & 0,0482 & 1,70 & $-0,0767$ & $-1,10$ \\
\hline tpte y telecom * ETT & 0,3281 & 14,40 & $-0,1430$ & $-2,38$ & 0,5087 & 12,29 & $-1,0715$ & $-8,78$ \\
\hline educacion * ETT & 0,0459 & 1,39 & 0,3992 & 5,66 & 0,2463 & 7,61 & $-0,2721$ & $-3,53$ \\
\hline sanidad * ETT & $-0,1252$ & $-1,05$ & 0,7477 & 3,38 & 0,2871 & 3,02 & 0,1410 & 0,72 \\
\hline servicios * ETT & 0,3797 & 9,65 & 0,3176 & 3,54 & 0,4089 & 8,24 & $-0,1547$ & $-1,31$ \\
\hline contrato indefinido & 0,1288 & 22,09 & $-0,0523$ & $-3,62$ & 0,2582 & 23,51 & $-0,2597$ & $-10,27$ \\
\hline inmigrante * C.I. & 0,0044 & 0,08 & 0,3465 & 2,80 & $-0,0612$ & $-0,53$ & 0,0935 & 0,36 \\
\hline cualificacion medio - alto ${ }^{*}$ C.I. & 0,1691 & 6,60 & 0,2063 & 3,82 & 0,0358 & 0,61 & 0,1507 & 1,31 \\
\hline cualificacion medio ${ }^{*}$ C.I. & 0,1162 & 6,18 & $-0,0326$ & $-0,88$ & 0,0497 & 1,97 & $-0,1368$ & $-2,81$ \\
\hline sector público ${ }^{*}$ C.I. & $-0,0011$ & $-0,04$ & $-0,8028$ & $-12,38$ & 0,1671 & 2,62 & $-0,7504$ & $-6,79$ \\
\hline educacion * C.I. & 0,0038 & 0,07 & $-0,3549$ & $-3,98$ & $-0,1851$ & $-4,75$ & $-0,2078$ & $-2,75$ \\
\hline tamaño $101-250 *$ C.I. & 0,0950 & 9,63 & $-0,2177$ & $-8,46$ & $-0,0899$ & $-4,35$ & $-0,1621$ & $-3,29$ \\
\hline tamaño $251-500 *$ C.I. & $-0,0640$ & $-2,67$ & $-0,1638$ & $-3,25$ & $-0,0886$ & $-3,06$ & $-0,1309$ & $-1,79$ \\
\hline tamaño $501-1000 *$ C.I. & 0,1275 & 3,97 & $-0,1562$ & $-1,82$ & 0,1649 & 5,39 & $-0,5720$ & $-7,81$ \\
\hline Constante & 6,0740 & 255,71 & $-0,5475$ & $-9,47$ & 5,8878 & 145,38 & $-1,2009$ & $-11,24$ \\
\hline Observaciones & 32.322 & & & & 11.828 & & & \\
\hline Log likelihood & $-12.434,68$ & & & & $-4.815,01$ & & & \\
\hline
\end{tabular}

Grupo de referencia: trabajador de una empresa dedicada al comercio de menos 10 empleados, no inmigrante, con baja cualificación, de menos de 6 meses de antigüedad en el puesto de trabajo, no contratado por una ETT, sin trabajo fijo. 
Cuadro 7. Descomposiciones de Blinder-Oaxaca

\begin{tabular}{|c|c|c|c|c|c|}
\hline & & $\begin{array}{c}\text { Diferencias en las } \\
\text { características }\end{array}$ & $\begin{array}{l}\text { Diferencias en el } \\
\text { retorno a la media }\end{array}$ & $\begin{array}{l}\text { Diferencias en el } \\
\text { retorno a la } \\
\text { varianza }\end{array}$ & Total \\
\hline \multicolumn{6}{|c|}{ España } \\
\hline \multirow{2}{*}{$\mathrm{MCO}$} & & 0.03982 & 0.13084 & & 0.17067 \\
\hline & en porcentaje & $23.33 \%$ & $76.67 \%$ & & $100 \%$ \\
\hline Modelo censurado & & 0.04053 & 0.13122 & 0.00216 & 0.17391 \\
\hline estándar* & en porcentaje & $23.31 \%$ & $75.45 \%$ & $1.24 \%$ & $100 \%$ \\
\hline Modelo censurado con & & 0.03377 & 0.14231 & -0.00279 & 0.17329 \\
\hline $\begin{array}{c}\text { estimación de la } \\
\text { varianza }\end{array}$ & en porcentaje & $19.49 \%$ & $82.12 \%$ & $-1.61 \%$ & $100 \%$ \\
\hline \multicolumn{6}{|c|}{ Andalucía } \\
\hline \multirow{2}{*}{$\mathrm{MCO}$} & & 0.04974 & 0.12196 & & 0.17170 \\
\hline & en porcentaje & $28.97 \%$ & $71.03 \%$ & & $100 \%$ \\
\hline Modelo censurado & & 0.05228 & 0.12532 & -0.00140 & 0.17619 \\
\hline estándar & en porcentaje & $29.67 \%$ & $71.13 \%$ & $-0.80 \%$ & $100 \%$ \\
\hline Modelo censurado con & & 0.05094 & 0.12940 & -0.00325 & 0.17710 \\
\hline $\begin{array}{c}\text { estimación de la } \\
\text { varianza }\end{array}$ & en porcentaje & $28.77 \%$ & $73.07 \%$ & $-1.84 \%$ & $100 \%$ \\
\hline \multicolumn{6}{|c|}{ Cualificación Alta } \\
\hline \multirow{2}{*}{$\mathrm{MCO}$} & & 0.11184 & 0.04138 & & 0.15322 \\
\hline & en porcentaje & $72.99 \%$ & $27.01 \%$ & & $100 \%$ \\
\hline Modelo censurado & & 0.12228 & 0.04454 & -0.00540 & 0.16141 \\
\hline estándar & en porcentaje & $75.75 \%$ & $27.59 \%$ & $-3.35 \%$ & $100 \%$ \\
\hline Modelo censurado con & & 0.11810 & 0.04700 & -0.00626 & 0.15884 \\
\hline $\begin{array}{c}\text { estimación de la } \\
\text { varianza }\end{array}$ & en porcentaje & $74.35 \%$ & $29.59 \%$ & $-3.94 \%$ & $100 \%$ \\
\hline \multicolumn{6}{|c|}{ Cualificación Baja } \\
\hline \multirow{2}{*}{$\mathrm{MCO}$} & & 0.03247 & 0.15174 & & 0.18421 \\
\hline & en porcentaje & $17.63 \%$ & $82.374 \%$ & & $100 \%$ \\
\hline Modelo censurado & & 0.03340 & 0.15563 & -0.00285 & 0.18618 \\
\hline estándar & en porcentaje & $17.94 \%$ & $83.59 \%$ & $-1.53 \%$ & $100 \%$ \\
\hline Modelo censurado con & & 0.02925 & 0.15568 & 0.00077 & 0.18570 \\
\hline $\begin{array}{c}\text { estimación de la } \\
\text { varianza }\end{array}$ & en porcentaje & $15.75 \%$ & $83.84 \%$ & $0.41 \%$ & $100 \%$ \\
\hline
\end{tabular}

* Descomposición hecha de acuerdo con la fórmula (10). La misma descomposición es usada para el modelo censurado con estimación de la varianza y para cada uno de los subgrupos: Andalucía, Cualificación Alta y Cualificación Baja. 
Gráfico 1. Histograma y Función de Densidad (Kernel) de los salarios masculinos en España.

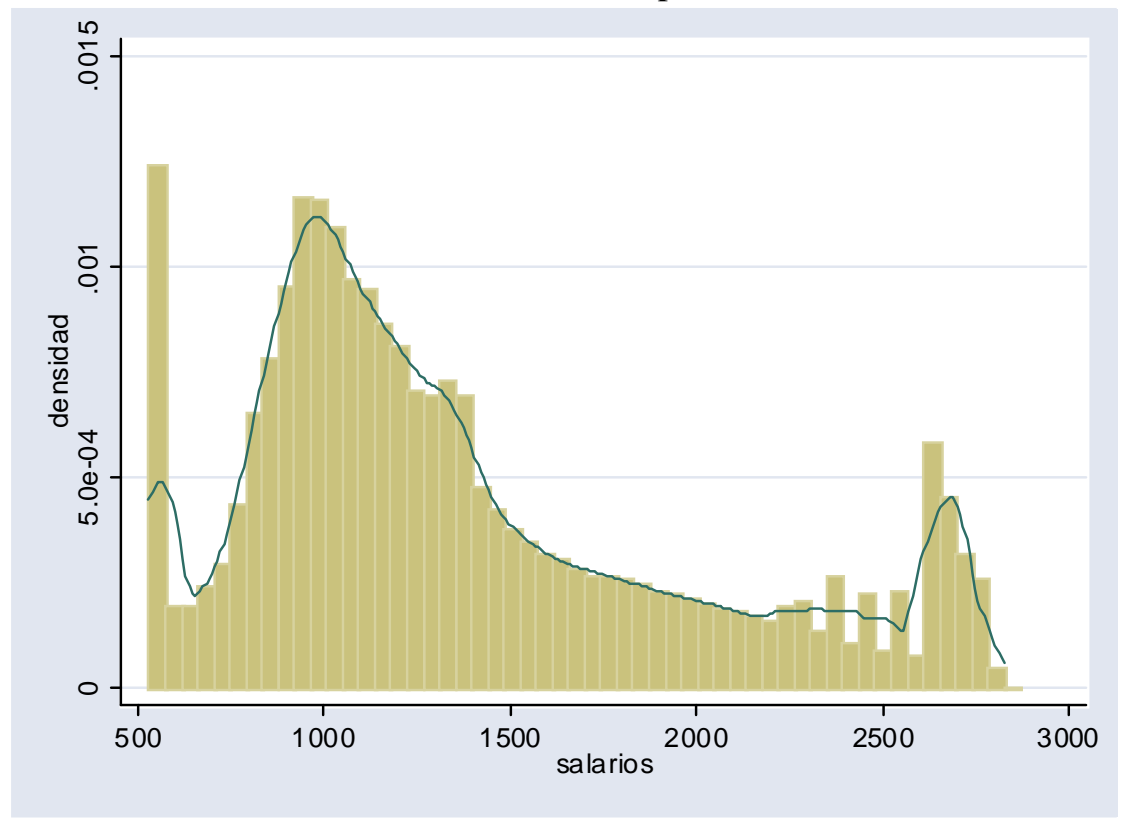

Gráfico 2. Histograma y Función de Densidad (Kernel) de los salarios femeninos en España.

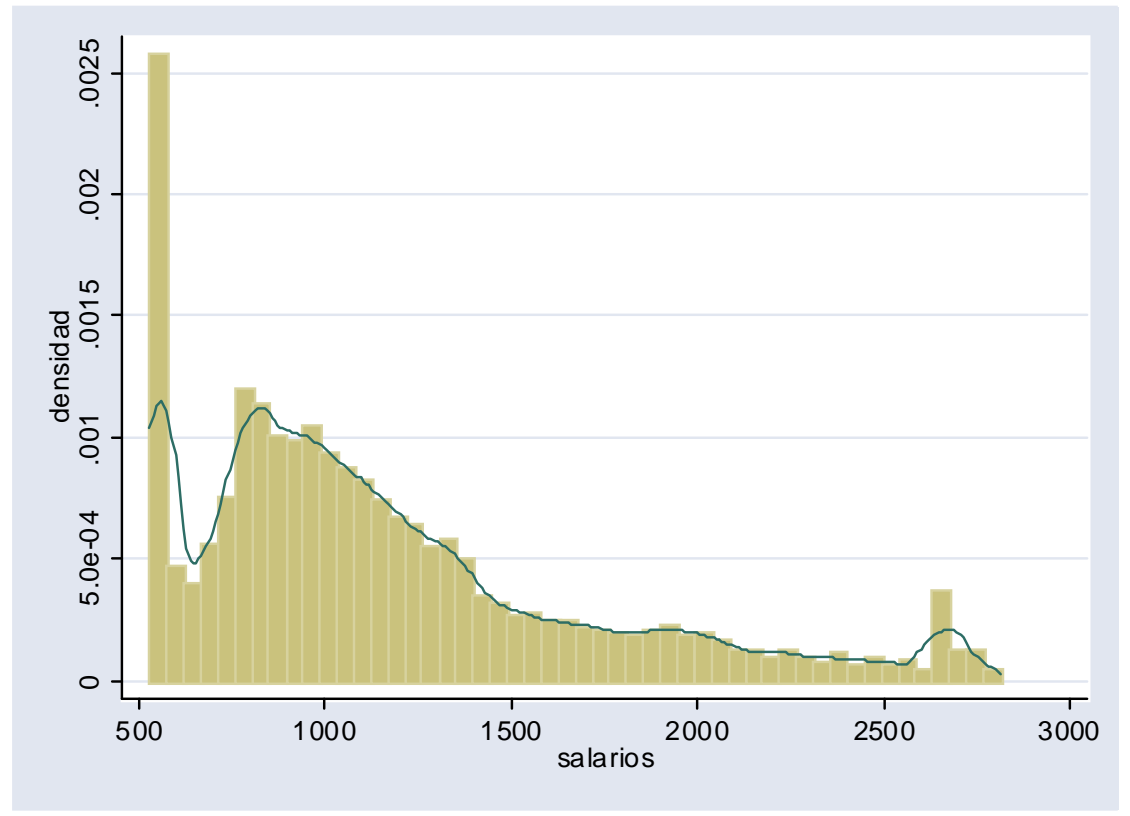


Gráfico 3. Histograma y Función de Densidad (Kernel) de los salarios masculinos en Andalucía.

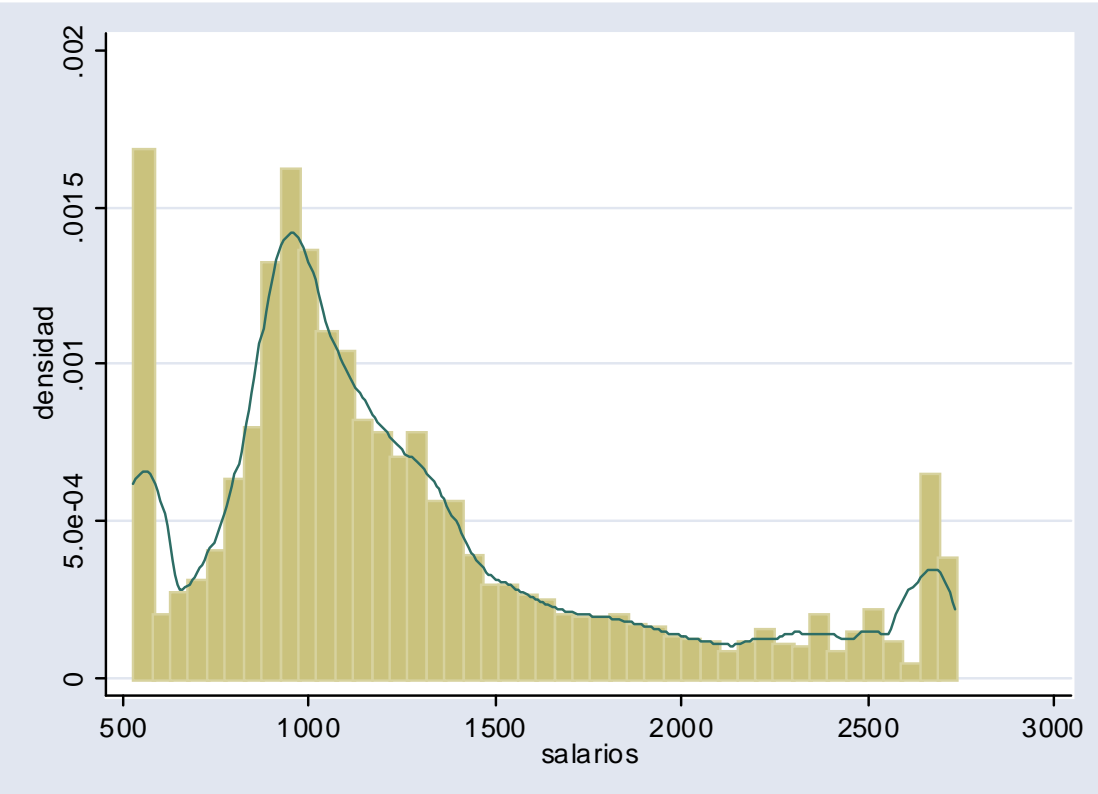

Gráfico 4. Histograma y Función de Densidad (Kernel) de los salarios femeninos en Andalucía.

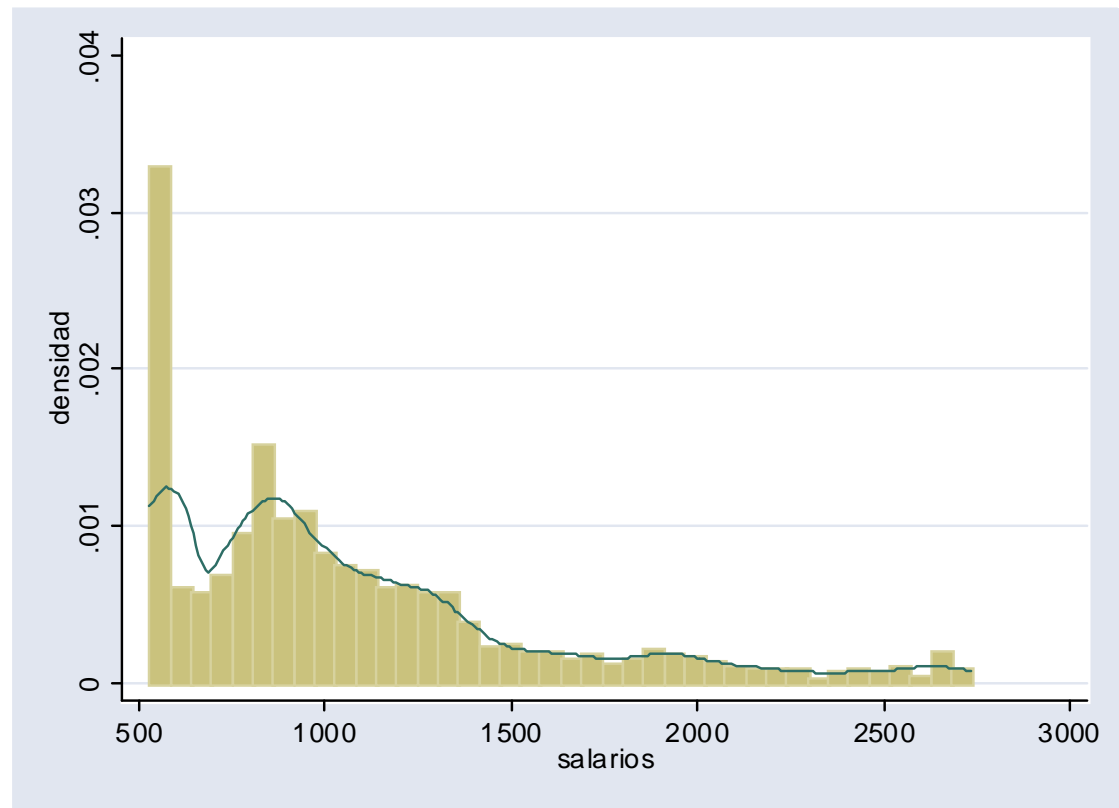




\section{Apéndice: Variables utilizadas}

La construcción y definición de las variables que hemos utilizado en el análisis empírico son las siguientes:

- Salarios mensuales: Están basados en la base de cotización mensual de cada individuo en euros de 2003 deflactados por el IPC de cada comunidad autónoma. Pueden estar censurados, lo cual se determina teniendo en cuenta las bases de cotización mínima y máxima para cada grupo de cotización establecidos por el régimen general de la Seguridad Social. Sólo se consideran los salarios de contratos a tiempo completo.

- Edad: Consideramos trabajadores con edades comprendidas entre 16 y 65 años.

- Inmigrante: Con esta variable están registrados los individuos inmigrantes de fuera de la Unión Europea, excluyendo además los provenientes de otros países industrializados como EEUU, Canadá, Noruega, Suiza y Japón.

- Cualificación: Tomando como base los once grupos de cotización establecidos por la Seguridad Social definimos cinco grupos, atendiendo a la cualificación requerida para el puesto de trabajo. Los grupos son los siguientes: Grupo de tarifa Alta (ingenieros, licenciados, ingenieros técnicos, peritos, ayudantes titulados y asimilados), grupo de tarifa media - alta (jefes administrativos y de taller, ayudantes no titulados), grupo de tarifa media (oficiales administrativos y subalternos), grupo de tarifa media baja (auxiliares administrativos, oficiales de $1^{\mathrm{a}}$ y $2^{\circ}$ ) y baja (oficiales de tercera y especialistas, peones y trabajadores menores de dieciocho años).

- Sector productivo: En la estimación empírica distinguimos once sectores reagrupados a partir de la clasificación a dos códigos de la CNAE. Hemos excluido el sector "agrícola" porque los salarios de este sector no son comparables con el resto teniendo en cuenta que muchos de los trabajadores en este sector son trabajadores autónomos o de temporada. La variable“sector público" incluye sólo administrativos, personal de seguridad y defensa. 
- Tamaño de la empresa: La muestra de estimación cubre empresas de hasta mil empleados, dado que para tamaños mayores la base de datos no es representativa. Se distinguen por el tamaño cinco tipos de empresas: de hasta diez trabajadores, de once a cincuenta, de cincuenta y uno a cien, de ciento uno a doscientos cincuenta, de doscientos cincuenta y uno a quinientos y de quinientos uno a mil trabajadores.

- Antigüedad: Esta variable se ha generado a partir del mes y año de alta del individuo en el contrato de trabajo estudiado, así como del mes y año en el momento en que se extrae la base de datos. Por tanto, estamos hablando de antigüedad en el trabajo actual, ya que, si existe más de un contrato en la misma empresa, estamos considerándolos todos como parte de una misma relación laboral. 